\title{
Global landscape of replicative DNA polymerase usage in the human genome
}

Eri Koyanagi ${ }^{1, \#}$, Yoko Kakimoto ${ }^{1, \#}$, Fumiya Yoshifuji ${ }^{2, \#}$, Toyoaki Natsume ${ }^{3,4, \dagger}$, Atsushi Higashitani ${ }^{2}$, Tomoo Ogi ${ }^{5}$, Antony M. Carr ${ }^{6}$, Masato T. Kanemaki ${ }^{3,4}$, Yasukazu Daigaku ${ }^{1,2, *}$

${ }^{1}$ Frontier Research Institute for Interdisciplinary Sciences, Tohoku University, Sendai, Japan

${ }^{2}$ Graduate School of Life Sciences, Tohoku University, Sendai, Japan

${ }^{3}$ National Institute of Genetics, Research Organization of Information and Systems (ROIS), Mishima, Japan

${ }^{4}$ Department of Genetics, The Graduate University for Advanced Studies (SOKENDAI), Mishima, Japan

${ }^{5}$ Research Institute of Environmental Medicine, Nagoya University, Nagoya, Japan

${ }^{6}$ Genome Damage and Stability Centre, School of Life Sciences, University of Sussex, Falmer, United Kingdom, BN1 9RQ

"These authors contributed equally to this work.

${ }^{\dagger}$ Present address: Research Center for Genome \& Medical Sciences, Tokyo Metropolitan Institute of Medical Science, Tokyo, Japan

*Corresponding author (e-mail: daigaku@tohoku.ac.jp) 


\section{Abstract}

The division of labour among DNA polymerase underlies the accuracy and efficiency of replication. However, the roles of replicative polymerases have not been directly established in human cells. We developed polymerase usage sequence (Pu-seq) in HCT116 cells and mapped Pole and Pol $\alpha$ usage genome wide. The polymerase usage profiles show Pole synthesises the leading strand and Pol $\alpha$ contributes mainly to lagging strand synthesis. Combining the Pole and Pol $\alpha$ profiles, we accurately predict the genome-wide pattern of fork directionality plus zones of replication initiation and termination. We confirm that transcriptional activity contributes to the pattern of initiation and termination and, by separately analysing the effect of transcription on both co-directional and converging forks, demonstrate that coupled DNA synthesis of leading and lagging strands in both codirectional and convergent forks is compromised by transcription. Polymerase uncoupling is particularly evident in the vicinity of large genes, including the two most unstable common fragile sites, FRA3B and FRA3D, thus linking transcription-induced polymerase uncoupling to chromosomal instability.

\section{Introduction}

Accurate DNA replication underlies stable genetic inheritance and is essential in all eukaryotic organisms. In humans, the loss of replication fidelity is responsible for genetic changes that cause both inherited syndromes and somatic diseases, including cancer. There are 16 different DNA polymerases in eukaryotes and the fidelity and efficiency of their synthetic activities are distinct ${ }^{1}$. The division of labour among these polymerases is, therefore, a primary factor in determining the accuracy of genome duplication. In both the budding and fission yeasts, three DNA polymerases: Pol $\delta$, Pol $\varepsilon$ and Pol $\alpha$, have been demonstrated to be required for genome replication and are thus termed replicative polymerases. To start all canonical replication events, primase initiates a short RNA primer that is subsequently extended for 10-20 nucleotides by Pol $\alpha$. On the leading strand the bulk of DNA synthesis is subsequently completed by Pole. On the lagging strand, where synthesis is by necessity discontinuous, Pol $\delta$ takes over from Pol $\alpha$ to extend the synthesis up to 100 to 200 bp, generating the Okazaki fragment.

The roles of the replicative polymerases were first established in budding yeast from the mutational bias caused by altered (mutagenic) replicative polymerases in the vicinity of an efficient replication origin, where replication directionality could be predicted ${ }^{2,3}$. Using a similar mutational bias approach in fission yeast, the role of Pol $\delta$ in lagging strand synthesis was shown to be conserved. Using a mutated Pole that is prone to introducing ribonucleotides (rNMPs) into DNA it was also shown, using strandspecific alkali sensitivity, that the role of Pol $\varepsilon$ in synthesising the leading strand was similarly conserved ${ }^{4}$. To expand the analysis of polymerase usage genome-wide, the locations of the increased levels of rNMPs incorporated by individual mutated DNA polymerases (Pol $\alpha$, Pol $\delta$ or Pol $\varepsilon$ ) were identified by whole genome sequencing ${ }^{5-7}$. These data provided direct evidence that the bulk of leading strand synthesis is performed by Pole, while that of the lagging strand was the responsibility of Pol $\alpha$ and Pol$\delta$. Consistent with these in vivo reports, the roles of budding yeast replicative polymerases have similarly been demonstrated by in vitro studies that reconstituted the replisome with purified factors ${ }^{8,9}$. 
In addition to confirming the division of labour among replicative DNA polymerase, the genome-wide data of replicative polymerase usage also provided highly detailed and discriminatory information about replication fork dynamics. For example, genomic sites with an increased probability of either replication initiation or termination associate with reciprocal changes in leading and lagging strand polymerase usage. By calculating relative changes (differential derivatives) of the profiles of the individual replicative polymerases, the population percentage of replication initiation and termination events were globally measured. This approach identified replication initiation sites, plus their probability of initiation (efficiency), at unpreceded resolution in both budding and fission yeast. It also provided an estimation of the probability of termination across the genome ${ }^{5,10}$. The accuracy of the methodology was exemplified by the fact that initiation sites identified in budding yeast correspond with the known sequence-specificity of replication origins. In fission yeast, the initiation sites correlated closely with AT richness ${ }^{11}$. Indeed, Monte Carlo simulation of replication fork dynamics based solely on the predicted distribution of the origin recognition complex calculated from the genomic AT content produced a profile of fork dynamics that was strikingly similar to the experimental data ${ }^{12}$.

The profiles of leading and lagging strand DNA polymerase usage can also be directly converted into replication fork directionality (RFD), which represents the proportion of leftward or rightward moving forks at each genomic locus. Again, the accuracy of these data has been verified by multiple studies. For example, mathematical analysis of the RFD data derived from polymerase usage has been used to predict replication timing (RT) across entire chromosomes. The resulting data is superimposable on experimental RT data derived from measured DNA copy number ${ }^{5}$. Given the precision, quantitative accuracy and the concordance with previous measurement of replication fork dynamics, the identification of polymerase usage provides a rational approach to explore genome replication globally in other eukaryotic organisms.

In contrast to lower eukaryotes, the division of labour among replicative polymerases in metazoan cells remains to be addressed. Although human Pol $\alpha$ and Pol $\delta$ have been shown to be required for the synthesis of both leading and lagging strands in reconstituted SV40 replication systems ${ }^{13}$, the usage of replicative polymerases during genomic DNA replication has not been directly characterised. To elucidate the division of labour among replicative polymerases in human cells, and to analyse the profile of replication forks at high resolution, we set out to track the usage of leading and lagging strand DNA polymerases across the human genome. In order to track synthesis by replicative DNA polymerases, we followed the equivalent logic of polymerase usage sequencing (Pu-seq, also known as HydEn-seq) in the yeasts and exploited alleles of replicative DNA polymerases that incorporate an excess of rNMP into DNA during synthesis ${ }^{5-7}$ (Fig. 1a).

Using the near-diploid colon cancer cell line HCT116 we successfully produced genome-wide profiles of Pol $\varepsilon$ and Pol $\alpha$ usage that, respectively, reflected leading and lagging strand synthesis. By analysing these profiles we confirm that transcriptional activity influences replication initiation, demonstrate that 
fork directionality impacts termination close to transcription start sites and show that transcription can perturb the coupling of leading and lagging strand polymerases. Finally, we also show that, at several common fragile sites (CFS) expressed in HCT116 cells, a high level of uncoupled polymerase usage is apparent due to the local inhibition of leading strand DNA synthesis.

\section{Results}

\section{Construction of ribonucleotide-incorporating DNA polymerase mutant lines}

Ribonucleotides are normally incorporated by the replicative polymerases approximately 1:4000 incorporation events. The mutated alleles of replicative polymerases used in yeast to map polymerase usage significantly increase this rate of incorporation. Nonetheless, such rNMPs are rapidly removed from duplex DNA by ribonucleotide excision repair (RER), which is initiated by the RNase H2 enzyme. Therefore, RNase H2 must be inactivated concomitantly with increased rNMP incorporation in order to map the distribution of rNMPs genome-wide. Unlike in yeast, RNase $\mathrm{H} 2$ is essential for growth of mammalian cells in the presence of $\mathrm{p} 53^{14}$. Thus, we developed a HCT116 cell line where we could induce acute degradation of the largest RNase H2 subunit, RNASEH2A, by the addition of auxin. In this auxin-inducible degron (AID) system ${ }^{15}$ the target protein, RNASEH2A, is tagged with the minimal auxin-inducible degron tag (mAID) and an auxin receptor protein from rice (OsTIR) is expressed. Treatment of the cells with the plant hormone 3-indole-acetic acid (IAA) promotes an interaction between OsTIR1 (an Cullin E3 ubiquitin ligase component) and the mAID-tagged RNASEH2A. Recruiting mAID tagged RNASEH2A to the Cullin E3 ligase by the addition of IAA induced ubiquitylation-dependent degradation of the targeted protein (Fig. 1b left).

To generate mutant alleles of the POLD1, POLE1 and POLA1 genes (encoding the catalytic subunits of Pol $\delta$, Pol $\varepsilon$ and Pol $\alpha$ respectively) that are predicted to promote increased rNMP incorporation during synthesis we aligned the highly conserved amino acid sequence of their catalytic sites with the corresponding yeast polymerases. This identified POLD1-L606G, POLE1-M630F and POLA1-Y865F as equivalent mutations to those exploited in budding and fission yeasts. Using CRISPR-Cas9-mediated homology-directed repair, we attempted to generate bi-allelic mutations in the RNASEH2A-degron HCT116 cells (Fig. 1c). As a result, POLE1-M630F and POLA1-Y865F mutations were independently introduced into both alleles of the corresponding gene. The POLD1-L606G mutation was, however, only introduced into the one allele, even after repetitive trials. We also observed that ectopically expressing the POLD1-L606G gene does not provide the essential function of the POLD1 gene.

Using the biallelic mutant cell lines for Pole and Pol $\alpha$, in addition to relevant control cell lines, we examined whether RNASEH2A degradation causes increased levels of rNMP in the DNA. Genomic DNA was extracted, treated with alkaline (which preferentially hydrolyses the phosphate-backbone 3' of the incorporated rNMPs) and the extent of fragmentation was examined by running the denatured samples on agarose gels ${ }^{5,6}$. For the POLE1-M630F and POLA1-Y865F cell lines, increased levels of 
small DNA fragments were observed upon RNASEH2A degradation when compared control cell lines. This indicates that rNMPs are incorporated at appreciably higher levels by the mutated Pol $\varepsilon$ and Pol $\alpha$ (Fig. 1de). However, rNMP incorporation in the POLA1-Y865F cell line was limited in comparison to the POLE1-M630F cell line. We therefore adapted the POLA1-Y865F cell line to the recently developed AID2 system that exploits the highly specific binding of a mutated version of OsTIR (F74G) with an auxin analogue, 5-Ph-IAA ${ }^{16}$. As expected, more efficient degradation of RNASEH2A was observed (Fig. 1b right) and considerably increased levels of incorporated rNMP were evident in the POLA1-Y865F cell line when compared to controls (Fig. 1f).

\section{Mapping polymerase usage across the genome}

To map usage of Pole and Pol $\alpha$, DNA was prepared from the relevant cell lines 48 hours after IAA/5Ph-IAA addition and small alkaline-cleaved single stranded-DNA (ssDNA) fragments $(<2 \mathrm{~kb})$ were collected and used to produce libraries for Illumina sequencing. Approximately 200 million paired-end reads were obtained for each cell line and the positions of 5' ends were mapped to either the Watson or Crick strands. The 5' ends represent the rNMP positions, which were scored in 1-kb bins across the genome. The relative ratio of reads for Pol $\varepsilon$ and Pol $\alpha$ mutants, when compared to those of control lines, provides scores representative of relative usage of these polymerases (Fig. 2a). Along the chromosome coordinates, a reciprocal relationship was evident between the profiles of Pol $\varepsilon$ and Pol $\alpha$ on the same strand. Similarly, a reciprocal relationship was evident between the profiles of same polymerase when comparing the Watson with the Crick strands. These patterns of polymerase profiles are evident across the genome, consistent with the primary roles of Pol $\varepsilon$ in leading strand and Pol $\alpha$ in lagging strand synthesis (Fig. 2b). Importantly, two independent experiments were confirmed to yield nearly identical polymerase profiles (Supplementary fig. 1). These results demonstrate the roles of Pole and Pol $\alpha$ are conserved between yeasts and humans, although replicon size (the region replicated from a single replication initiation site) is quite different: $30-50 \mathrm{~kb}$ in yeasts vs. several hundred $\mathrm{kb}$ to 1-1.5 $\mathrm{Mb}$ in humans. Interestingly, visual inspection of the profiles shows that the typical enrichment of either leading or lagging strand synthesis was not evident in some areas of the genome. These regions exclusively locate at heterochromatic late replicating segments (71 sites across the genome, Supplementary fig. 2abe). This suggests that DNA replication is regulated differently in these regions (see below).

The profiles of leading and lagging strand polymerases provide two direct and independent measurements of the proportions of replication forks moving either rightward or leftward at each location across the genome. We therefore calculated replication fork direction profiles independently from either the Pole $\left(\mathrm{RFD}^{\varepsilon)}\right.$ or Pol $\alpha\left(\mathrm{RFD}^{\alpha}\right)$ data and compared these with OK-seq replication directionality data that we calculated from sequencing data ${ }^{17,18}$ using the identical algorithm (RFD ${ }^{\mathrm{OK}}$; for details see materials and methods). Visual inspection indicates that the overall trends of $\mathrm{RFD}^{\varepsilon}, \mathrm{RFD}^{\alpha}$ and $\mathrm{RFD}^{\mathrm{OK}}$ are highly similar (Supplementary fig. 3ab), albeit with the Pu-seq derived data showing 
lower amplitude peaks. $\mathrm{RFD}^{\alpha}$ is more similar to $\mathrm{RFD}^{\mathrm{OK}}$ than to $\mathrm{RFD}^{\varepsilon}$ (Supplementary fig. 3c), indicating that the profile of Pol $\alpha$ captures signatures specific to lagging strand synthesis. Considering the minor differences between leading and lagging strand synthesis, we established a combined RFD profile from pooled Pole and Pol $\alpha$ Pu-seq data. Fluctuation outside of the local trends are notably reduced when compared to $\mathrm{RFD}^{\varepsilon}$ or $\mathrm{RFD}^{\alpha} \quad$ (Supplementary fig. 3a). Thus, combining both leading and lagging strand profiles improves precision as well as resolution of the replication fork profiles $\left(\mathrm{RFD}^{\varepsilon \mid \alpha}\right)$.

\section{Defining replication initiation regions from polymerase usage}

The division of polymerase labour between leading and lagging strands dictates that sites of frequent replication initiation manifest as reciprocal demarcations in Pol $\varepsilon$ and Pol $\alpha$ usage. Therefore, we defined an initiation parameter to represent the local activity of initiation events (Fig. 2c). Specifically, we calculate two independent initiation indices (Supplementary fig. 4ab), one from the Pole data (Ini where Pol $\varepsilon$ synthesis increases towards the 3' on the Watson strand and Pol $\varepsilon$ decreases towards the 5' on the Crick strand) and a second from the Pol $\alpha$ data (Ini ${ }^{\alpha}$, where Pol $\alpha$ synthesis decreases towards the 3' on the Watson strand and Pol $\alpha$ increases towards the 5' on the Crick strand). These two independent indices can be plotted separately (Supplementary fig. 4ab) or combined into a more accurate and constrained cumulative initiation index (Ini ${ }^{\varepsilon \mid \alpha}$, Fig. 2cd). The two replicates of the Pu-seq experiments were used to generate two independent combined initiation indices (Supplementary fig. 4c). Positive peaks in the initiation index are interpreted as replication initiation sites and peak height as proportional to the population frequency of initiation. Approximately 12,000 initiation peaks were detected in each replicate. The concordance in the peak positions between the two replicates increases with increasing values of the initiation index; when all the peaks were taken into account, $67.2 \%$ of all peaks colocalise, whereas this number increased to $87.5 \%$ with top $50 \%$ of initiation peaks. (Supplementary fig. 4d). Since polymerase profiles were derived from asynchronous cells, this analysis detects initiation events during the entire $\mathrm{S}$ phase. Of note, the initiation index can also have negative values, which represents regions where termination of merging forks is frequent.

Previously mapped initiation sites in the human genome showed that a proportion localised to a few kilobases, whereas other mapped to 'initiation zones' of $\sim 10-50 \mathrm{~kb}^{17,19,20}$. In our initiation index profile, the width of positive peaks averaged $\sim 33 \mathrm{~kb}$ (replicate 1: 32.7kb, replicate 2: $33.1 \mathrm{~kb}$ ) with more than $20 \%$ above 50kb (Fig. 3a) confirming that initiation events in human cells cluster in zonal regions. The activity of initiation per zone size increases with size, up to approx. $70 \mathrm{~kb}$ (Supplementary fig. 4e) and, when the initiation index is plotted with high-resolution $\mathrm{RT}^{21}$, the zones consistently locate at local peaks of RT (Fig. 3b, Supplementary fig. S5). We note that initiation zones are present both in midlate RT regions (e.g. around 41Mb, $60 \mathrm{Mb}$ in Chr. 3 in Fig. 3b, locations marked by circles in Supplementary fig. S5) as well as early replicating regions. These data thus demonstrate that high probability initiation zones are not located primarily in early replicating regions, but exist in mid-late 
replication regions. This is contrary to the prevailing view that efficient initiation is the predominant determinant of early replicating regions and indicates that late-firing but efficient initiation zones exist across the human genome.

We also observed 71 regions of $\mathrm{Mb}$ length heterochromatic late replicating regions where clear peaks for initiation zones were not evident (supplementary fig. 2c). These defined the same regions noted above as having unusual fork direction profiles (Supplementary fig. S2a). The lack of defined initiation zones was consistent with the reported profile of high-resolution $\mathrm{RT}^{21}$, where defined initiation sites were not also observed within these regions (supplementary fig. 2d). This likely reflects that replication initiation occurs at random locations within late replicating heterochromatinic regions. This would also account for the equal frequency of leftward and rightward moving forks.

\section{Association of replication Initiation/termination with transcriptional activity}

A positional relationship between replication initiation and transcription has been highlighted by multiple studies using different techniques ${ }^{17,18,20,22}$. We therefore analysed how the distribution of transcription units influences the initiation and termination of replication forks in our Pu-seq derived data. The initiation index was aligned at transcription start sites (TSS) and transcription termination sites (TTS). The initiation index score increases in the vicinity of both TSS and TTS (Fig. 3c-e). High levels of gene expression (Fig. 3c) and increased gene length both correlated with increasing initiation index score (Fig. 3d), consistent with published OK-seq data from RPE-1 cells ${ }^{18}$. However, the higher resolution of the Pu-seq derived data shows that the peak of initiation localises $\sim 20 \mathrm{~kb}$ upstream of the TSS and $\sim 20 \mathrm{~kb}$ downstream of the TTS. We also note that initiation index shows a negative value throughout gene bodies, consistent with frequent termination in these regions (Fig. 3de).

We next aligned the Pu-seq-derived RFD data with TSS and TTS. At TSS and the 5' regions of genes we observe a significant bias of rightward moving forks. At the TTS and 3' regions of genes we observe a bias towards leftward moving forks (Fig. 3fg). Thus, as expected when initiation is biased towards TSS and TTS (and is largely absent from gene bodies), termination is increased within the gene body (Fig. 3h). Notably, these localised initiation and termination patterns are far more apparent for large genes. While the distribution of genes is a factor in the location of initiation and termination zones our result also demonstrate that there are many genes that are transcriptionally active but do not show replication initiation in the vicinity of their TSS and TTS. Plotting the extent of replication initiation at TSS/TTS against transcriptional activity, it is evident that most of the genes associated with replication initiation are transcriptionally active. However, transcriptionally active genes are not necessarily associated with replication initiation: approximately $20 \%$ of genes with $>$ average transcriptional activity show no evidence of initiation in the upstream of TSS (Supplementary fig. 6a). These data suggest that many transcriptionally active genes are passively replicated and transcriptional units do not account for all replication initiation. Furthermore, approximately $60 \%$ of initiation zones do not overlap 
with upstream regions of TSS or downstream regions of TTS (Supplementary fig. 6b).

It is evident that more forks travel from the initiation zones upstream of the TSS into the gene bodies (i.e. co-directional with transcription) than travel from the gene body through the TSS convergent to the direction of transcription. However, this is not absolute and we estimate for the top $50 \%$ of transcribed genes this equates to between 66-51\% co-directional fork and between $49-34 \%$ of forks that are convergent with transcription, which varies dependent on gene length (Fig. 3g). To separately visualise the dynamics of replication forks dependent on their orientation at any one locus, we calculated a separate 'fork index' for both rightward $\left(\mathrm{Fk}^{\mathrm{R}}\right)$ and leftward $\left(\mathrm{Fk}^{\mathrm{L}}\right)$ moving forks that independently represent their cumulative initiation and termination behaviours. These profiles can be interpreted as separate 'initiation indices' for rightward and leftward moving forks. Thus, fork initiation and termination are represented by positive or negative values (Fig. 4a). Visual inspection of genome-wide $\mathrm{Fk}^{\mathrm{R}}$ and $\mathrm{Fk}^{\mathrm{L}}$ profiles showed similar genomic profiles that are, as expected, congruent with the initiation index discussed above (Fig. $\mathbf{4 b}$ the experimental replicate in Supplementary fig. 7).

Aligning $\mathrm{Fk}^{\mathrm{R}}$ and $\mathrm{Fk}^{\mathrm{L}}$ at TSS allowed us to separately visualise the effect of transcription on forks that are either co-directional (CD) or convergent (CV) with transcription (Fig. 4cde). In these figures, leftward moving forks that initiate upstream of TSS - high peak of fork index - move away from the gene and the fork index thus declines slowly. However, leftward moving forks within the gene body are CV with transcription and showed dramatically decreased fork index downstream of TSS (Fig. 4def). This suggests that head-to-head transcription replication clashes slows fork processivity and increases termination events in this region. In the case of CD forks (rightward moving) the fork index profile at TSS is more complex: two lower peaks are evident in the vicinity of TSS. We interpret this as a combination of initiation and termination: i.e. a negative signal (fork termination) at and immediately upstream of TSS is embedded within strong positive signals derived from initiation events associated with the upstream $20 \mathrm{~kb}$. It should be noted as discussed above that not all transcriptionally active genes are associated with an increase in initiation upstream of TSS (Supplementary fig. 6a). In the transcriptionally active genes (> average) which do not show an associated increase of replication initiation at TSS, the fork index for CD forks drops sharply below zero at and immediately upstream of TSS ('a-1' in Supplementary fig. 6c), indicating that forks terminate in this region. As expected, the profile of genes that show association of initiation with the TSS resembles that of all active genes ('a2' in Supplementary fig. 6c, Fig. 4d). Thus, this mixed signal of fork termination and initiation manifested in fork index does not necessary represent the profile of individual loci. The combined pattern of CV and CD forks is consistent with the trend for fork initiation in both orientations $\sim 20 \mathrm{~kb}$ upstream of TSS, with the processivity of leftward moving (CV) forks being reduced immediately downstream of TSS (Fig. 4d top) and the processivity of rightward moving (CD) being reduced immediate upstream of TSS (Fig. 4dbottom). Taking gene length into account demonstrates that this effect is largely independent of gene length and manifests throughout the length of the transcription unit 
(Fig. 4e,f). This tendency is contrary to fork initiation, which increase with gene length, indicating that fork termination also commonly occurs in the vicinity of TSS. During transcription initiation RNA Polymerase II (RNAPII) promoter-proximal pausing is enriched in the immediate vicinity of TSS ${ }^{23}$. Thus RNAPII, directly or indirectly, likely causes an impediment to fork progression. In contrast, as predicted by the initiation index around TTS, both initiation and termination events are evident and fork orientation (co-directional or convergent) did not influence the profiles.

\section{Local genomic features at replication initiation sites.}

To investigate if specific sequence-based or chromatin-based features correlate with replication initiation sites, peak positions within initiation zones were computationally identified (yellow vertical lines in Fig. 2c bottom and Supplementary Fig. 2c) and those present in initiation zones of both experimental replicates selected. Using this dataset we examined if specific genomic elements are enriched at these loci. GC skew, AT skew and CpG islands were not enriched. Potential guanine quadruplex (G4) structures were modestly enriched at peaks of initiation index (Supplementary fig. 8a). For chromatin features the H2AZ histone variant and, to a lesser extent, trimethylated H3K27 (H3K27me3) were enriched at initiation index peaks (Supplementary fig. 8b). In contrast, trimethylated H3K36 (H3K36me3) tended to be excluded from initiation zones and moderately enhanced in flanking regions, likely because it is associated with gene bodies ${ }^{23}$. H3K4me3 was enriched $\sim 20 \mathrm{~kb}$ either side of the peak of initiation, consistent with its enrichment at TSS ${ }^{23}$ (Supplementary fig. 8c). Furthermore, analysing genomic status bin based on multiple chromatin profiles by using ChromHMM algorism ${ }^{24}$, initiation zones and TSS/TTS were shown to associate with distinct chromatin status (Supplementary fig. 8d). Thus, the chromatin features H2AZ and H3K27me3 positively correlate specifically with initiation sites, while H3K36me3 shows a minor anti-correlation.

To establish which of the features discussed above correlate best with initiation activity we partitioned each chromosome into $10 \mathrm{~kb}$-bins and used principal component analysis (PCA) to deconvolve genomic features in relation to replication initiation (Supplementary fig. 8e). This revealed that chromatin-based features (H2AZ and H3K27me3) correlated more closely with replication initiation than G4. As expected, H3K36me3, which is deposited in transcribed regions in an RNAPII-dependent manner (thus marking gene bodies), positioned oppositely to initiation. These data suggest that, combinatorially with transcription, chromatin status is crucial for shaping replication initiation.

\section{Uncoupling of leading and lagging strand polymerases}

Having two independent datasets that represent leading (Pole) and lagging (Pol $\alpha$ ) strand synthesis offers the opportunity to examine how well coupled DNA synthesis is throughout the genome. As expected, Pole and Pol $\alpha$ usage on the leading and lagging stands respectively, for forks moving in the same direction is notably similar. However, reproducible differences in Pol $\varepsilon$ and Pol $\alpha$ profiles are detected (e.g. around $26 \mathrm{Mb}$ on chromosome 6 in Fig. 5a, experimental replicate: Supplementary fig. S9). We 
interpret this as evidence of uncoupling between leading and lagging strand polymerases ${ }^{25}$. To quantify this, we calculated a separate coupling index (CI) for rightward and leftward moving forks to represent the bias toward either Pol $\varepsilon$ or Pol $\alpha$ usage (Fig. 5b). If both leading and lagging strand synthesis contribute equally to replication of the duplex DNA, CI $=0$. Positive values of CI represent a bias toward leading strand polymerase (Pole) while a negative value represent a bias toward lagging strand polymerase (Pol $\alpha)$.

Across the majority of genomic regions the CI remains close to zero, but at some loci it reproducibly deviates by $+/-0.25$, suggesting up to $25 \%$ biased usage of Pol $\varepsilon$ or Pol $\alpha$ occurs relatively frequently across the genome (Fig. 5cd). We observed a significant correlation between the two biological replicates (Supplementary fig. 10a). Interestingly, we observed a reciprocal pattern for CI between rightward and leftward moving forks (Fig. 5d, Supplementary Fig. 10b). This indicates an opposite bias for forks moving in the two directions is present. For example, at the histone-encoding gene clusters on chromosome 6, usage of Pole was overrepresented compared Pol $\alpha$ in the rightward forks and the opposite trend was observed in leftward forks (Fig. 5c, Supplementary fig. 9). This inverse correlation is conserved across the genome (replicate $1: r=-0.403 p<0.001$, replicate 2 : $r=-0.354 p<0.001$, Fig. 5d, Supplementary fig. 10b). We next examined if these CI fluctuations correlated with transcription. Aligning CI of co-directional (CD) and converging forks (CV) at TSS sites we observed that the CI of CD forks increased within gene bodies, whereas the CI of CV forks decreased (Fig. 5ef). This likely reflects the orientation of DNA polymerase movement and transcription: synthesis by forks moving in the same direction of RNAPII encounter problems that result in a bias toward Pole (suggesting lagging strand synthesis is impaired), while synthesis by forks moving in the opposite direction to RNAPII result in problems that result in a bias towards Pol $\alpha$ (suggesting that leading strand synthesis is impaired). Consequently, DNA synthesis toward opposite to transcription was shown to be impaired in both CD and CV forks (further discussed below).

The genome wide distribution of CI variation tends toward lower values (Fig. 5d, Supplementary fig. 10b). This suggests that leading strand synthesis is generally more susceptible to spontaneous impediment than lagging strand synthesis. The affected regions are not uniformly dispersed across the genome and tend to favour particular chromosomes (Fig. 6a). To identify regions associated with this phenomenon, we statistically identified 27 regions as CI outliner loci, where either rightward or leftward CI values diverged significantly from the population of chromosomal data in both biological replicates (Table 1). Among these, 16 loci (59.2\%) were associated with large genes. In all but one of these the $\mathrm{CI}$ was notably low $(\mathrm{CI}<-0.3)$ for forks converging with transcription. One explanation of these results is transcription stress due to head-to-head collisions becomes intense in a subset of large genes and consequently polymerase uncoupling occurs frequently due to an impediment of leading strand DNA synthesis. However, it should not be excluded that an alternative engagement of other polymerases, such as Pol $\delta$ during replication restart ${ }^{26,27}$ causes the low CI values. 
In three of the uncoupling regions (Table 1) CI values were significantly negative for both rightward (CD) and leftward (CV) moving forks. This indicates perturbation of leading strand DNA synthesis on both strands of the duplex (Fig. 6bc). Two of these three loci are positioned within the FRA3B and FRA16D common fragile sites (CFSs) that are highly expressed in many cell lines, including HCT116 ${ }^{28}$. The low CI loci did not correlate with replication initiation or termination zones, suggesting that specific impediments to leading strand polymerases occur at these regions. Comparing our data with published End-seq experiments in HCT116 ${ }^{29}$, the low CI loci do not overlap with hotspots of double strand breaks (DSBs). Thus, we propose that uncoupling of replicative polymerase is separate feature of at least a subset of CFS and can contribute to chromosome rearrangement in a manner independent of DSBs.

\section{Discussion}

By locating the positions of incorporated rNMP by mutated DNA polymerases in human HCT116 cells we have characterised genome-wide usage of the replicative polymerases Pol $\varepsilon$ and Pol $\alpha$. The profiles of Pol $\varepsilon$ and Pol $\alpha$ usage on either the Watson or Crick strands are strikingly reciprocal. While Pol $\alpha$ primes both leading and lagging strand synthesis, the number of lagging strand priming events vasty exceed that of the leading strand. Thus, our data demonstrate that human Pol $\varepsilon$ and Pol $\alpha$ contribute to leading and lagging strand DNA synthesis, respectively, across the genome. These results establish that the roles of Pol $\varepsilon$ and Pol $\alpha$, and by implication Pol $\delta$, are conserved between human and yeasts ${ }^{5-7}$. Our attempts to characterise Pol $\delta$ were not successful because the POLD1-L606G or POLD1-L606M mutants (predicted to elevate rNMP-incorporation) could not be isolated.

\section{Replication initiation}

The Pol $\varepsilon$ and Pol $\alpha$ profiles for the Watson and Crick strands represent the population average of fork directionality. Our data provide four independent measures of fork directionality. By combining these, we calculated genome wide replication fork directionality (RFD) profiles with high resolution. Sites of frequent replication initiation are, by definition, characterised by changes between the leading and lagging strand polymerases on both the Watson and Crick strands. We therefore calculated an initiation index for each location (1-kb bin), defining locations characteristic of initiation as those where all four polymerase profiles exhibited the expected difference from its neighbouring location. Approximately 12,000 peaks of positive initiation index scores were identified in each biological replicate, a number comparable of those identified by Ori-SSDS for mouse cells $(11000-13000)^{30}$. The intra peak distance average was $230 \mathrm{~kb}$, slightly larger than previous estimates (160-190 kb) ${ }^{31}$. The average width of the zones of positive initiation index scores was (33 kb), comparable to OK-seq (mean $30 \mathrm{~kb}$, range 6-150 $\mathrm{kb}^{17}$ ) and optical replication mapping (mean $32 \mathrm{~kb}^{32}$ ). These data confirm that initiation in human cells is zonal. 


\section{Transcription and initiation dynamics}

Consistent with previous reports, we observed a correlation between replication initiation and TSS and TTS of transcribed genes. The high resolution of our data allowed us to unambiguously map the centre of these initiation zones $\sim 20 \mathrm{~kb}$ upstream and downstream of the TSS and TTS, respectively. While the correlation between TSS/TTS and initiation was highly significant, our data also demonstrate that 20\% of genes that are transcribed above the average level do not show increase in initiation enrichment close to TSS/TTS sites and that $60 \%$ of initiation zones do not localise in the vicinity of TSS/TTS. By examining chromatin features for correlation with initiation zones, we identified H2AZ and, to a lesser extent H3K27me3, as peaking with initiation. Modifications directly associated with transcription, such as H3K4me3 (TSS associated ${ }^{23}$ ) and H3K36me3 (gene body associated), showed separate and distinct profiles. These appear to reflect the proximity of the modification at TSS or gene bodies and are likely not causative correlations. We speculate that H2AZ and H3K27me3 contribute to configuring an accessible local environment for initiation zones, in which $\mathrm{H} 2 \mathrm{AZ}$ and/or other chromatin features recruit ORC or pre-RC components ${ }^{33}$.

\section{Uncoupling of leading and lagging strands}

A novel aspect of our approach is that having strand specific data for two independent polymerases allowed us to define a fork coupling index (CI - see Fig. 5b) that quantifies how well leading and lagging strand synthesis are coupled during fork progression. Surprisingly, a reproducible bias toward either Pol $\varepsilon$ (leading) or Pol $\alpha$ (lagging) strand polymerases is relatively common and ranges up to $25 \%$ at some loci. The fluctuation of the CI for human cells appear much greater than that previously observed in yeasts ${ }^{5,10}$ and, unlike in yeasts where polymerase bias is associated with initiation or termination, CI fluctuations are prominent in regions not associated with these phenomena. Molecular events that might generate CI fluctuation include the inhibition of DNA synthesis to generate strandspecific polymerisation defects or DNA synthesis by other polymerases. Thus, CI fluctuation likely reflect multiple mechanisms that can occur either within ongoing replication forks or after fork passage or collapse.

Studies using E. coli or budding yeast protein or Xenopus extracts ${ }^{34-36}$ showed that, upon uncoupling of the helicase and leading strand polymerase, the helicase slows to encourage recoupling. In vitro analysis using budding yeast proteins suggests that Pol $\delta$ recouples synthesis. In our analysis, this would manifest as a negative CI (bias towards Pol $\alpha$ ). Alternatively, if DNA polymerase usage at uncoupled regions is determined by noncanonical polymerases not intrinsic to the fork, the CI would fluctuate toward either positive or negative values, depending on whether leading or lagging stand synthesis was influenced. While noncanonical polymerase usage has mainly been associated with DNA damage tolerance, there is increasing evidence for such phenomena under unperturbed conditions, particularly in mammalian cells ${ }^{37-41}$. Our data thus raise the important question of how extensively the unanticipated level of the plasticity of DNA polymerase usage underpins replication of the human genome. Of note we 
demonstrate that inhibition of the leading strand polymerase is particularly evident in FRA3B and FRA16D in the absence of exogenous replication stress.

\section{The influence of transcription on replication fork dynamics}

By separately analysing the polymerase usage data associated with rightward (Watson; leading strand template, Crick; lagging strand template) and leftward (Watson; lagging strand template, Crick; leading strand template) moving forks we can evaluate the effect of transcription on the dynamics of either convergent or co-directional replication forks (see Fig. 4). This established that, at immediately upstream of TSS, co-directional forks are impaired - most likely when they encounter paused RNAPII, which is frequent around transcription initiation sites. Similarly, convergent forks moving from the gene body showed evidence of impaired processivity immediately downstream of TSS. A similar distinction between converging and co-directional forks was not evident at TTS, where both appear to be impeded upstream of TTS within the gene body. Thus, we reveal distinct fates for codirectional and convergent forks at transcription start sites. To our knowledge, no evidence of perturbation of replication forks at TSS or promoter regions has previously been reported.

The accumulation of RNAPII pausing has been shown to be a phenomenon associated with cancerprone situations ${ }^{42,43}$. The consequent increase in replication-transcription conflicts, such as we demonstrate here, may underlie this association. We note that the impairment to fork dynamics calculated for a single direction $\left(\mathrm{Fk}^{\mathrm{L}}\right.$ and $\mathrm{Fk}^{\mathrm{R}}$ ) does not appear in our initiation indices (Ini ${ }^{\varepsilon}$, Ini ${ }^{\alpha}$ and $\mathrm{Ini}^{\varepsilon \mid \alpha}$ ) because initiation indices are defined to describe fork dynamics across both orientations. Thus, by establishing the effects of transcription on both the initiation index and fork index, our analysis separately detects the impediment to fork progression at TSS or the termination of two merging forks, which we show occur at different preferential locations relative to genes ${ }^{42,43}$.

Consistent with clashes between replication and transcriptional machineries, we observed increased coupling index fluctuations in gene bodies, implying transcription-replication conflicts cause frequent polymerase uncoupling. Separating out the effects on convergent and co-directional forks, the block to DNA synthesis tends to manifest where the non-transcribed strand is used as template DNA. One potential explanation is that, when DNA is transcribed, R-loop structures can form behind RNAPII. These would lead to transient regions of ssDNA on the non-transcribed strand. ssDNA is chemically less stable than dsDNA and more susceptible to damaging agents and some DNA-modifying enzymes ${ }^{44}$. It is also of note that transcription coupled repair eliminates DNA damage specifically on the transcribed strand. As a result, DNA damage on the non-transcribed strand may become relatively more influential in perturbing DNA synthesis. Whatever the underlying causes, this transcriptional effect is particularly manifested in large genes, which may reflect the fact that transcription must proceed beyond the end of $\mathrm{S}$ phase $\mathrm{e}^{45}$. One possibility is that the accumulation of positive supercoiling ahead of, and negative supercoiling behind, RNAPII not only pauses replication forks but also enhances the level of 
transcription-induced blocks, perhaps by inducing excessive fork rotation, which previous studies in budding yeast have found to generates post-replicative stress ${ }^{46}$.

In summary, Pu-seq in human cells provides a powerful and straightforward methodology to explore DNA polymerase usage and replication fork dynamics. We show here that data produced from Pu-seq are highly consistent with those from other methods such as high resolution repli-seq, OK-seq. The two independent datasets required for Pu-seq provide four separate measures of replication dynamics, allowing predictions of replication fork movement, initiation and termination with great accuracy. Exploiting this we show that transcription influences circa $40 \%$ of initiation events and contributes significantly to replication fork uncoupling and subsequent genome instability. Pu-seq will thus provide a useful tool for examining DNA replication by manipulating specific genetic changes in the HCT116 cell lines we have developed, or by constructing the system in different cell lines harbouring distinct developmental or genetic backgrounds.

\section{Materials and Methods}

\section{Cell culture}

All cell lines are derived from HCT116 cells (ATCC, \#CCL-247) and are listed in Table 2. Cells were cultured in McCoy's 5A, supplemented with 10\% FBS (Gibco, \#10437-028), 2 mM L-glutamine, 100 $\mathrm{U} / \mathrm{ml}$ penicillin and $100 \mu \mathrm{g} / \mathrm{ml}$ streptomycin at $37^{\circ} \mathrm{C}$ in $5 \% \mathrm{CO}_{2}$. IAA (Nakarai tesque \#19119-61), 5Ph-IAA (BioAcademia \#30-003) and auxinole (BioAcademia \#30-001) were dissolved in DMSO to create a $500 \mathrm{mM}$ stock solution (stored at $-20^{\circ} \mathrm{C}$ ) and further diluted with media to an appropriate concentration and added directly (1:25 v/v) to the culture medium to achieve the working concentration (IAA $500 \mu \mathrm{M}$, 5-Ph-IAA $400 \mathrm{nM}$, auxinole $100 \mu \mathrm{M}$ ). Doxycycline was dissolved in water to create a 1 $\mathrm{mg} / \mathrm{ml}$ stock solution (stored at $\left.-20^{\circ} \mathrm{C}\right)$ and added directly $(1: 1000 \mathrm{v} / \mathrm{v})$ to the culture media to achieve working solution $(1 \mu \mathrm{g} / \mathrm{ml})$.

\section{Generation of polymerase mutants}

Cas9 protein (Alt-R® S.p. HiFi Cas9 Nuclease V3, \#1081060) and tracrRNA (Alt-R® CRISPR-Cas9 tracrRNA, \#1072533) and crRNA (Alt-R® CRISPR-Cas9 crRNA, custom production, Table 2) were purchased from IDT (Integrated DNA Technologies, USA). Guide RNA (gRNA) was formed by mixing equimolar amounts ( $50 \mu \mathrm{M})$ of tracrRNA and crRNA in duplex buffer (IDT), heating to $96^{\circ} \mathrm{C}$ for $5 \mathrm{~min}$, and cooling on the benchtop to room temperature. $61 \mu \mathrm{M}$ of Cas9 and gRNA were mixed at a ratio of 2:3 and incubated for $0.5-1 \mathrm{hr}$. Following RNP formation, $1.5 \mu \mathrm{l}$ of RNP and $1.5 \mu \mathrm{l}$ of $36 \mu \mathrm{M}$ single stranded oligodeoxynucleotide (ssODN) template (listed in Table 2) was added into $1 \times 10^{5}$ cells in 12 $\mu \mathrm{l}$ of R buffer in Neon ${ }^{\circledR}$ transfection system $10 \mu \mathrm{L}$ Kit (Invitrogen, \#MPK1096) and the cell suspension applied to Neon ${ }^{\circledR}$ transfection system with 10 Neon tips. Following electroporation cells were immediately suspended into $500 \mu \mathrm{l}$ of media and Alt-R ${ }^{\mathrm{TM}}$ HDR Enhancer V2 (IDT, \#10007921) to 
achieve $20 \mu$ Mwas added. 3 day after transfection, 300-3000 cells were plated in $10 \mathrm{~cm}$ dishes for colony formation. 96 single colonies were picked into wells of a 96 well plate. After replicating the clones into another 96 well plate cells were incubated for 2-4 days. The cells on one plate was subjected to genotyping by PCR and those on the other plate was stored at $-80^{\circ} \mathrm{C}$ with Bam banker DIRECT medium (Nippon Genetics, CS-06-001).

\section{Generation of mAID- Clover-tagged RNASEH2A cell line}

Cells were transfected with CRISPR-Cas9 and donor plasmids (Table 2) using FuGENE HD Transfection Reagent (Promega, \#E2311) in a 6-well plate following the manufacturer's instructions. Two days after transfection, cells were plated in $10 \mathrm{~cm}$ dishes and selected with antibiotics. Selected clones were isolated and confirmed as previously described ${ }^{47}$.

The degradation of RNASEH2A cell line in AID or AID2 system.

For the AID system the +TetOsTIR mAID-Clover-tagged RNASEH2A cells (Table 2) were treated with doxycycline and auxinole ${ }^{47}$ for 24 hrs to induce OsTIR1 without background degradation and this medium was replaced with medium containing doxycycline and IAA. The cells were incubated for a further indicated period. For AID2 system, where OsTIR1(F74G) is constitutively expressed, +OsTIR(F74G) mAID-Clover-tagged RNASEH2A cells (Table 2) were treated with 5Ph-IAA for the indicated period.

\section{DNA extraction, alkaline treatment and library preparation}

$2 \times 10^{7}$ cells were harvested by centrifugation and genomic DNA was prepared using Blood \& Cell Culture DNA Midi Kit 100/G Genomic-tips (Qiagen \#13343). To examine alkaline degradation $3 \mu \mathrm{g}$ of DNA was treated with $0.3 \mathrm{M} \mathrm{NaOH}$ at $55^{\circ} \mathrm{C}$ for $2 \mathrm{hr}$ in $15 \mu \mathrm{l}$. The reaction was stopped by adding $3 \mu \mathrm{l}$ of $1 \mathrm{M}$ Tris-HCl (pH7.5). $1 \mu \mathrm{l}$ of this solution was subjected to TapeStation RNA ScreenTape Analysis (Agilent \#5067-5576, \#5067-5577, \#5067-5578) to detect ssDNA. For library preparation $25 \mu \mathrm{g}$ of genomic DNA was alkali treated in $0.3 \mathrm{M} \mathrm{NaOH}$ at $55^{\circ} \mathrm{C}$ for $2 \mathrm{hr}$, then loaded onto a $1.5 \%$ agarose gel and run for $1 \mathrm{hr} 40 \mathrm{~min}$ at $100 \mathrm{~V}$. The gel was stained with acridine orange (final concentration $5 \mu \mathrm{g} / \mathrm{ml}$ ) for $2 \mathrm{hr}$ at room temperature with gentle shaking followed by overnight destaining in water. Fragments of 300-2000 bp were excised from the gel and isolated with a gel-extraction kit (Macherey-Nagel, NucleoSpin Gel and PCR Clean-up, \#740609). Library preparation was performed as previously described $^{5,48}$. Libraries were 150-bp paired-end (PE) sequenced on an Illumina Hiseq X platform (Macrogen, Tokyo, Japan).

\section{Analysis of Polymerase Usage}

For each sample approx. 200 million PE read were obtained. Raw reads were aligned to GRCh38 using Bowtie2 (version 2.3.5). Those which aligned to multiple genomic locations with the same mismatch scores (AS and XS scores as outputted by Bowtie2) were excluded using a custom Perl script: sam-dup- 
align-exclude-v2.pl (available at the GitHub site: https://github.com/yasukasu/sam-dup-align-exclude). The position of the 5' end of each R1 read (which corresponds to the 5' end of ssDNA hydrolysed by alkali treatment) was determined, and the number of reads in 1-kb bins across the genome were counted separately for the Watson and Crick strands using a custom Perl script: pe-sam-to-bincount.pl (available at the GitHub site: https://github.com/yasukasu/sam-to-bincount). This generated the four datasets for the analysis of one polymerase. In case of Pole: at the chromosome coordinate $\mathrm{x}, \mathrm{N}_{\mathrm{w}}{ }^{\varepsilon}(\mathrm{x})$, is the count for RNASEH2-mAID POLE1-M630F on the Watson strand; $\mathrm{N}_{\mathrm{c}}^{\varepsilon}(\mathrm{x})$ is the count for RNASEH2-mAID POLE1-M630F on the Crick strand; $\mathrm{N}_{\mathrm{w}}{ }^{+}(\mathrm{x})$ is the count for RNASEH2-mAID $\mathrm{POL}^{+}$on the Watson strand; $\mathrm{N}_{\mathrm{c}}^{+}(\mathrm{x})$ is the count for RNASEH2-mAID $\mathrm{POL}^{+}$on the Crick strand.

The datasets were normalised using the total number of reads: e.g. $N^{\prime}{ }_{w}{ }^{\varepsilon}(x)=N_{w}{ }^{\varepsilon}(x) / \sum N_{w}{ }^{\varepsilon}$ for the Pole mutant on the Watson strand (where $\mathrm{N}$ ' indicates normalisation). These normalised data were used to calculate relative polymerase usage: e.g. $\mathrm{E}_{\mathrm{w}}(\mathrm{x})=\mathrm{N}{ }_{\mathrm{w}}{ }^{\varepsilon}(\mathrm{x}) / \mathrm{N}{ }_{\mathrm{w}}{ }^{+}(\mathrm{x})$ for usage of Pole on the Watson strand; $\mathrm{E}_{\mathrm{c}}(\mathrm{x})=\mathrm{N}_{{ }_{c}}{ }^{\varepsilon}(\mathrm{x}) / \mathrm{N}_{\mathrm{c}}{ }^{+}(\mathrm{x})$ for usage of Pol $\varepsilon$ on the Crick strand. The equivalent analysis was performed to yield usage of Pol $\alpha$ on Watson and Crick strand: $A_{w}(x)$ and $A_{c}(x)$. When these data were plotted or used for further analysis (below) they were smoothed using moving average of $2 m+1$, where $m$ is an arbitrary number the value of which is given in the relevant context. Thus, the data point for each bin is an average of $2 \mathrm{~m}+1$ bins: the point of origin and the $m$ bins either side.

\section{Initiation index and fork index}

The difference between each neighbouring data point of polymerase usage was calculated as $\Delta \mathrm{E}_{\mathrm{w}}(\mathrm{x})$, $\Delta \mathrm{E}_{\mathrm{c}}(\mathrm{x}), \Delta \mathrm{A}_{\mathrm{w}}(\mathrm{x})$ and $\Delta \mathrm{A}_{\mathrm{c}}(\mathrm{x})$ with $\mathrm{E}_{\mathrm{w}}(\mathrm{x}), \mathrm{E}_{\mathrm{c}}(\mathrm{x}), \mathrm{A}_{\mathrm{w}}(\mathrm{x})$ and $\mathrm{A}_{\mathrm{c}}(\mathrm{x})$ which were smoothed using the value $\mathrm{m}$ $=30$ (genome-wide plot, Fig. 2c, Fig3b ,Fig4b, Fig. 5c and Fig. 6bc) or $m=7$ (the plot of averages or heat map, Fig. 3c-e and Fig. 4d,e). These differential data were further smoothed by application of a moving average with value $m=15$ (genome-wide plot) or $m=7$ (the plot of averages or heat map). At each location where all four polymerase profiles exhibit consistent patterns for initiating bidirectional replication forks $\left(\Delta \mathrm{E}_{\mathrm{w}}(\mathrm{x})>0 \cap \Delta \mathrm{E}_{\mathrm{c}}(\mathrm{x})<0 \cap \Delta \mathrm{A}_{\mathrm{w}}(\mathrm{x})<0 \cap \Delta \mathrm{A}_{\mathrm{c}}(\mathrm{x})>0\right)$ or patterns consistent with the merging of two forks $\left(\Delta \mathrm{E}_{\mathrm{w}}(\mathrm{x})<0 \cap \Delta \mathrm{E}_{\mathrm{c}}(\mathrm{x})>0 \cap \Delta \mathrm{A}_{\mathrm{w}}(\mathrm{x})>0 \cap \Delta \mathrm{A}_{\mathrm{c}}(\mathrm{x})<0\right)$ an initiation index was defined as: $\operatorname{Ini}(\mathrm{x})=\Delta \mathrm{E}_{\mathrm{w}}(\mathrm{x})-\Delta \mathrm{E}_{\mathrm{c}}(\mathrm{x})-\Delta \mathrm{A}_{\mathrm{w}}(\mathrm{x})+\Delta \mathrm{A}_{\mathrm{c}}(\mathrm{x})$. This data was subjected to Z-score normalisation (mean $=0$, standard deviation $=1$ ) and $\mathrm{Z}(0)$ were subtracted to maintain the original + or - information, which represent increased levels of replication initiation and termination in the cell population respectively. Fork index was similarly calculated, but separately for rightward and leftward moving forks $\left(\mathrm{Fk}^{\mathrm{R}}\right.$ and $\left.\mathrm{Fk}^{\mathrm{L}}\right)$. For example, locations of rightward fork initiation are where $\Delta \mathrm{E}_{\mathrm{w}}(\mathrm{x})>0 \cap \Delta \mathrm{A}_{\mathrm{c}}(\mathrm{x})>0$. Similarly, positions of rightward moving fork termination are where $\Delta \mathrm{E}_{\mathrm{w}}(\mathrm{x})<0 \cap \Delta \mathrm{A}_{\mathrm{c}}(\mathrm{x})<0$. At these positions $\mathrm{Fk}$ was calculated as $F^{R}(x)=\Delta E_{w}(x)+\Delta A_{c}(x)$ to give the fork index of rightward moving forks. The equivalent was performed for leftward moving forks: location initiation is where $\Delta \mathrm{E}_{\mathrm{c}}(\mathrm{x})<0 \cap \Delta \mathrm{A}_{\mathrm{w}}(\mathrm{x})<0$ and termination where $\left.\Delta \mathrm{E}_{\mathrm{c}}(\mathrm{x})>0 \cap \Delta \mathrm{A}_{\mathrm{w}}(\mathrm{x})>0\right)$. The index for these locations was calculated as $\mathrm{Fk}^{\mathrm{L}}(\mathrm{x})$ $=-\mathrm{dE}_{\mathrm{c}}(\mathrm{x})-\mathrm{dA} \mathrm{A}_{\mathrm{w}}(\mathrm{x})$. These data were subjected to Z-scores normalisation and $\mathrm{Z}(0)$ subtracted. 


\section{RFDs from Pu-seq and Ok-seq}

RFD from polymerase usage were calculated by subtraction of polymerase profiles typical of leftward moving fork signals from rightward moving fork signals ${ }^{17}$. When using only Pol $\varepsilon$ usage data, RFD $^{\varepsilon}=$ $\left(E_{w}(x)-E_{c}(x)\right) /\left(E_{w}(x)+E_{c}(x)\right)$. When using only Pol $\alpha$ usage data, RFD ${ }^{\alpha}=\left(-A_{w}(x)+A_{c}(x)\right) /\left(A_{w}(x)+\right.$ $\left.A_{c}(x)\right)$. When using data from both polymerases, RFD ${ }^{\varepsilon \mid \alpha}=\left(E_{w}(x)-E_{c}(x)-A_{w}(x)+A_{c}(x)\right) /\left(E_{w}(x)+E_{c}(x)\right.$ $\left.+A_{w}(x)+A_{c}(x)\right)$. To calculate RFD from Ok-seq data, raw sequence read data from OK-seq experiments were obtained from the NCBI Sequence Read Archive (RPE1 ${ }^{18}$ : SRX4036932, GM06990 ${ }^{17}$ : SRX1427548, $\mathrm{HeLa}^{17}$ : SRX1424656) and mapped and counted using the same pipelines as used for the Pu-seq data. Okazaki fragment counts on Watson and Crick strands were defined as $\mathrm{OK}^{\mathrm{W}}(\mathrm{x})$ and $\mathrm{OK}^{\mathrm{C}}(\mathrm{x})$ and the RFD was calculated as: $\mathrm{RFD}^{\mathrm{OK}}=\left(\mathrm{OK}^{\mathrm{C}}(\mathrm{x})-\mathrm{OK}^{\mathrm{W}}(\mathrm{x})\right) /\left(\mathrm{OK}^{\mathrm{C}}(\mathrm{x})+\mathrm{OK}^{\mathrm{W}}(\mathrm{x})\right)^{17}$. The RFD datasets from Pu-seq and Ok-seq were further smoothed by application of a moving average where $m=3$. To convert $\mathrm{RFD}^{\varepsilon \mid \alpha}$ to rightward fork proportion, the range between values at -3 standard deviation and +3 standard deviation, which covers $99.7 \%$ of data, were converted to 0 to $100 \%$.

\section{Coupling index}

The data of polymerase usage: $E_{w}(x), E_{c}(x), A_{w}(x)$ and $A_{c}(x)$ were smoothed using the value $m=30$ (genome-wide or 2D plot, Fig. 5c,d, Fig. 6a-c) or $\mathrm{m}=7$ (the plot of averages or heat map, Fig. 5e,f). To establish a separate coupling index (CI) for both leftward and rightward forks, the lagging strand profile was subtracted from the leading strand profile. For rightward moving forks: $\mathrm{CI}^{\mathrm{R}}=\left(\mathrm{E}_{\mathrm{w}}(\mathrm{x})-\mathrm{A}_{\mathrm{c}}(\mathrm{x})\right) /\left(\mathrm{E}_{\mathrm{w}}(\mathrm{x})\right.$ $\left.+A_{c}(x)\right)$, for leftward moving forks: $C I^{L}=\left(E_{c}(x)-A_{w}(x)\right) /\left(E_{c}(x)+A_{w}(x)\right)$.

\section{RNA-seq data analsys}

Extraction of total RNA from HCT116 +TetOsTIR cells was performed by using Monarch ${ }^{\circledR}$ Total RNA Miniprep Kit (New England Biolabs, Inc, \#T2010S). cDNA library construction using TruSeq Stranded Total RNA Library Prep Kit with Ribo-Zero Human (Illumina \#RS-122-2201) and sequencing on an Illumina NovaSeq6000 platform was performed by Macrogen (Tokyo, Japan). Approx. 21 million pairend reads were sequenced. Raw sequenced reads were aligned to GRCh38 using STAR (version 2.7.3a, https://github.com/alexdobin/STAR). Annotations of transcript units in the human genome (version 94) were retrieved from the Ensembl Genome Brower (http://www.ensembl.org) and those which are categorised as transcript support level 1 or 2 and Esembl/Havana-merged transcripts (i.e. high probability of correct annotation) were chosen for inclusion in the alignment target set. To obtain data of fragments per Kilobase of exon per Million fragments mapped (FPKM), bam files of mapped reads was analysed using Cufflinks (http://cufflinks.cbcb.umd.edu/).

\section{Statistics and reproducibility.}

Two biological replicates were obtained for datasets of Pole and Pol $\alpha$ and both were used for all the analysis in this study. Data from one replicate are presented in Figs. 1-6: data from the second replicate 
showed excellent agreement and where relevant is shown in supplementary data.

Data availability

All data, including raw sequencing reads and source data for graphs in Figs. 1-6, are publicly available after publication . Custom scripts are available upon request from the corresponding authors.

\section{Acknowledgements}

This work was supported by: JST PRESTO grant (JPMJPR18K7) to YD; JSPS KAKENHI grants (JP16H06151, JP17K19336, JP20H03233 and JP21K19203) to YD; (JP20H05396 and JP21H04719) to MTK; Naito foundation, Takeda Science Foundation, Astellas foundation for research on metabolic disorders and NIG Collaborative Research Program grants (3B2016, 3A2017, 83A2019 and 81A2021) to YD; Wellcome Trust grant (110047/Z/15/Z) to AMC.

\section{Author contributions}

Y.D. conceived the study. Y.D. M.T.K. and A.M.C. designed the experimental approaches. Y.D designed informatics approaches. E.K., Y.K., F.Y., T.N., A.H., T.O. and Y.D. performed experiments and their analysis. Y.D. performed computational analysis. Y.D wrote the manuscript. A.M.C, M.T.K. and T.O. edited the manuscript

\section{Competing interests}

The authors declare no competing financial interests

\section{Materials \& Correspondence}

Should be addressed to Y.D. 


\section{References}

$1 \quad$ Lange, S. S., Takata, K. \& Wood, R. D. DNA polymerases and cancer. Nat Rev Cancer 11, 96110, doi:10.1038/nrc2998 (2011).

2 Pursell, Z. F., Isoz, I., Lundstrom, E. B., Johansson, E. \& Kunkel, T. A. Yeast DNA polymerase epsilon participates in leading-strand DNA replication. Science 317, 127-130, doi:10.1126/science.1144067 (2007).

3 Nick McElhinny, S. A., Gordenin, D. A., Stith, C. M., Burgers, P. M. \& Kunkel, T. A. Division of labor at the eukaryotic replication fork. Mol Cell 30, 137-144, doi:10.1016/j.molcel.2008.02.022 (2008).

4 Miyabe, I., Kunkel, T. A. \& Carr, A. M. The major roles of DNA polymerases epsilon and delta at the eukaryotic replication fork are evolutionarily conserved. PLoS Genet 7, e1002407, doi:10.1371/journal.pgen.1002407 (2011).

5 Daigaku, Y. et al. Aglobal profile of replicative polymerase usage. Nat Struct Mol Biol 22, 192198, doi:10.1038/nsmb.2962 (2015).

6 Clausen, A. R. et al. Tracking replication enzymology in vivo by genome-wide mapping of ribonucleotide incorporation. Nat Struct Mol Biol 22, 185-191, doi:10.1038/nsmb.2957 (2015). Reijns, M. A. M. et al. Lagging-strand replication shapes the mutational landscape of the genome. Nature 518, 502-506, doi:10.1038/nature14183 (2015).

Yeeles, J. T. P., Janska, A., Early, A. \& Diffley, J. F. X. How the Eukaryotic Replisome Achieves Rapid and Efficient DNA Replication. Mol Cell 65, 105-116, doi:10.1016/j.molcel.2016.11.017 (2017).

9 Georgescu, R. E. et al. Mechanism of asymmetric polymerase assembly at the eukaryotic replication fork. Nat Struct Mol Biol 21, 664-670, doi:10.1038/nsmb.2851 (2014).

10 Zhou, Z. X., Lujan, S. A., Burkholder, A. B., Garbacz, M. A. \& Kunkel, T. A. Roles for DNA polymerase delta in initiating and terminating leading strand DNA replication. Nat Commun 10, 3992, doi:10.1038/s41467-019-11995-z (2019).

11 Vazquez, E. \& Antequera, F. Replication dynamics in fission and budding yeasts through DNA polymerase tracking. Bioessays 37, 1067-1073, doi:10.1002/bies.201500072 (2015).

12 Kelly, T. \& Callegari, A. J. Dynamics of DNA replication in a eukaryotic cell. Proc Natl Acad Sci U S A 116, 4973-4982, doi:10.1073/pnas.1818680116 (2019).

13 Waga, S. \& Stillman, B. The DNA replication fork in eukaryotic cells. Annu Rev Biochem 67, 721-751, doi:10.1146/annurev.biochem.67.1.721 (1998).

14 Reijns, M. A. et al. Enzymatic removal of ribonucleotides from DNA is essential for mammalian genome integrity and development. Cell 149, 1008-1022, doi:10.1016/j.cell.2012.04.011 (2012).

15 Natsume, T., Kiyomitsu, T., Saga, Y. \& Kanemaki, M. T. Rapid Protein Depletion in Human Cells by Auxin-Inducible Degron Tagging with Short Homology Donors. Cell Rep 15, 210-218, doi:10.1016/j.celrep.2016.03.001 (2016). 
16 Yesbolatova, A. et al. The auxin-inducible degron 2 technology provides sharp degradation control in yeast, mammalian cells, and mice. Nat Commun 11, 5701, doi:10.1038/s41467-02019532-z (2020).

17 Petryk, N. et al. Replication landscape of the human genome. Nat Commun 7, 10208, doi:10.1038/ncomms10208 (2016).

18 Chen, Y. H. et al. Transcription shapes DNA replication initiation and termination in human cells. Nat Struct Mol Biol 26, 67-77, doi:10.1038/s41594-018-0171-0 (2019).

19 Gilbert, D. M. In search of the holy replicator. Nat Rev Mol Cell Biol 5, 848-855, doi:10.1038/nrm1495 (2004).

20 Langley, A. R., Graf, S., Smith, J. C. \& Krude, T. Genome-wide identification and characterisation of human DNA replication origins by initiation site sequencing (ini-seq). Nucleic Acids Res 44, 10230-10247, doi:10.1093/nar/gkw760 (2016).

21 Zhao, P. A., Sasaki, T. \& Gilbert, D. M. High-resolution Repli-Seq defines the temporal choreography of initiation, elongation and termination of replication in mammalian cells. Genome Biol 21, 76, doi:10.1186/s13059-020-01983-8 (2020).

22 Besnard, E. et al. Unraveling cell type-specific and reprogrammable human replication origin signatures associated with G-quadruplex consensus motifs. Nat Struct Mol Biol 19, 837-844, doi:10.1038/nsmb.2339 (2012).

23 Chen, F. X., Smith, E. R. \& Shilatifard, A. Born to run: control of transcription elongation by RNA polymerase II. Nat Rev Mol Cell Biol 19, 464-478, doi:10.1038/s41580-018-0010-5 (2018).

24 Ernst, J. \& Kellis, M. ChromHMM: automating chromatin-state discovery and characterization. Nat Methods 9, 215-216, doi:10.1038/nmeth.1906 (2012).

25 Berti, M., Cortez, D. \& Lopes, M. The plasticity of DNA replication forks in response to clinically relevant genotoxic stress. Nat Rev Mol Cell Biol 21, 633-651, doi:10.1038/s41580020-0257-5 (2020).

26 Miyabe, I. et al. Polymerase delta replicates both strands after homologous recombinationdependent fork restart. Nat Struct Mol Biol 22, 932-938, doi:10.1038/nsmb.3100 (2015).

27 Naiman, K. et al. Replication dynamics of recombination-dependent replication forks. Nat Commun 12, 923, doi:10.1038/s41467-021-21198-0 (2021).

28 Hosseini, S. A. et al. Common chromosome fragile sites in human and murine epithelial cells and FHIT/FRA3B loss-induced global genome instability. Genes Chromosomes Cancer 52, 1017-1029, doi:10.1002/gcc.22097 (2013).

29 Tubbs, A. et al. Dual Roles of Poly(dA:dT) Tracts in Replication Initiation and Fork Collapse. Cell 174, 1127-1142 e1119, doi:10.1016/j.cell.2018.07.011 (2018).

30 Pratto, F. et al. Meiotic recombination mirrors patterns of germline replication in mice and humans. Cell 184, 4251-4267 e4220, doi:10.1016/j.cell.2021.06.025 (2021).

31 Chagin, V. O. et al. 4D Visualization of replication foci in mammalian cells corresponding to 
individual replicons. Nat Commun 7, 11231, doi:10.1038/ncomms11231 (2016).

32 Wang, W. et al. Genome-wide mapping of human DNA replication by optical replication mapping supports a stochastic model of eukaryotic replication. Mol Cell 81, 2975-2988 e2976, doi:10.1016/j.molcel.2021.05.024 (2021).

33 Long, H. et al. H2A.Z facilitates licensing and activation of early replication origins. Nature 577, 576-581, doi:10.1038/s41586-019-1877-9 (2020).

34 Guilliam, T. A. \& Yeeles, J. T. P. Reconstitution of translesion synthesis reveals a mechanism of eukaryotic DNA replication restart. Nat Struct Mol Biol 27, 450-460, doi:10.1038/s41594020-0418-4 (2020).

35 Graham, J. E., Marians, K. J. \& Kowalczykowski, S. C. Independent and Stochastic Action of DNA Polymerases in the Replisome. Cell 169, 1201-1213 e1217, doi:10.1016/j.cell.2017.05.041 (2017).

36 Sparks, J. L. et al. The CMG Helicase Bypasses DNA-Protein Cross-Links to Facilitate Their Repair. Cell 176, 167-181 e121, doi:10.1016/j.cell.2018.10.053 (2019).

37 Lange, S. S. et al. Dual role for mammalian DNA polymerase zeta in maintaining genome stability and proliferative responses. Proc Natl Acad Sci U S A 110, E687-696, doi:10.1073/pnas.1217425110 (2013).

38 Fernandez-Vidal, A. et al. A role for DNA polymerase theta in the timing of DNA replication. Nat Commun 5, 4285, doi:10.1038/ncomms5285 (2014).

39 Bergoglio, V. et al. DNA synthesis by Pol eta promotes fragile site stability by preventing underreplicated DNA in mitosis. J Cell Biol 201, 395-408, doi:10.1083/jcb.201207066 (2013).

40 Barnes, R. P., Hile, S. E., Lee, M. Y. \& Eckert, K. A. DNA polymerases eta and kappa exchange with the polymerase delta holoenzyme to complete common fragile site synthesis. DNA Repair (Amst) 57, 1-11, doi:10.1016/j.dnarep.2017.05.006 (2017).

41 Twayana, S. et al. Translesion polymerase eta both facilitates DNA replication and promotes increased human genetic variation at common fragile sites. Proc Natl Acad Sci U S A 118, doi:10.1073/pnas.2106477118 (2021).

42 Zhang, X. et al. Attenuation of RNA polymerase II pausing mitigates BRCA1-associated Rloop accumulation and tumorigenesis. Nat Commun 8, 15908, doi:10.1038/ncomms15908 (2017).

43 Shivji, M. K. K., Renaudin, X., Williams, C. H. \& Venkitaraman, A. R. BRCA2 Regulates Transcription Elongation by RNA Polymerase II to Prevent R-Loop Accumulation. Cell Rep 22, 1031-1039, doi:10.1016/j.celrep.2017.12.086 (2018).

44 Gomez-Gonzalez, B. \& Aguilera, A. Transcription-mediated replication hindrance: a major driver of genome instability. Genes Dev 33, 1008-1026, doi:10.1101/gad.324517.119 (2019).

45 Helmrich, A., Ballarino, M. \& Tora, L. Collisions between replication and transcription complexes cause common fragile site instability at the longest human genes. Mol Cell 44, 966977, doi:10.1016/j.molcel.2011.10.013 (2011). 
46 Schalbetter, S. A., Mansoubi, S., Chambers, A. L., Downs, J. A. \& Baxter, J. Fork rotation and DNA precatenation are restricted during DNA replication to prevent chromosomal instability. Proc Natl Acad Sci U S A 112, E4565-4570, doi:10.1073/pnas.1505356112 (2015).

47 Yesbolatova, A., Natsume, T., Hayashi, K. I. \& Kanemaki, M. T. Generation of conditional auxin-inducible degron (AID) cells and tight control of degron-fused proteins using the degradation inhibitor auxinole. Methods 164-165, 73-80, doi:10.1016/j.ymeth.2019.04.010 (2019).

48 Keszthelyi, A., Daigaku, Y., Ptasinska, K., Miyabe, I. \& Carr, A. M. Mapping ribonucleotides in genomic DNA and exploring replication dynamics by polymerase usage sequencing (Pu-seq). Nat Protoc 10, 1786-1801, doi:10.1038/nprot.2015.116 (2015).

49 Lay, F. D. et al. The role of DNA methylation in directing the functional organization of the cancer epigenome. Genome Res 25, 467-477, doi:10.1101/gr.183368.114 (2015).

50 Marsico, G. et al. Whole genome experimental maps of DNA G-quadruplexes in multiple species. Nucleic Acids Res 47, 3862-3874, doi:10.1093/nar/gkz179 (2019). 
a

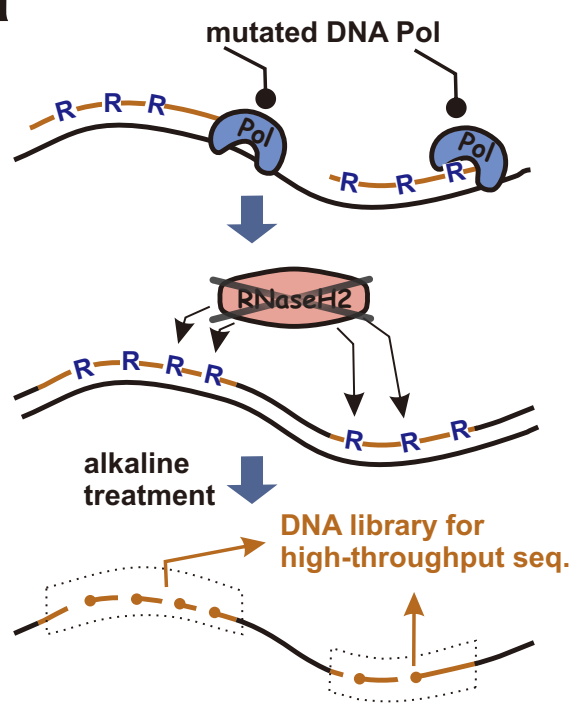

b

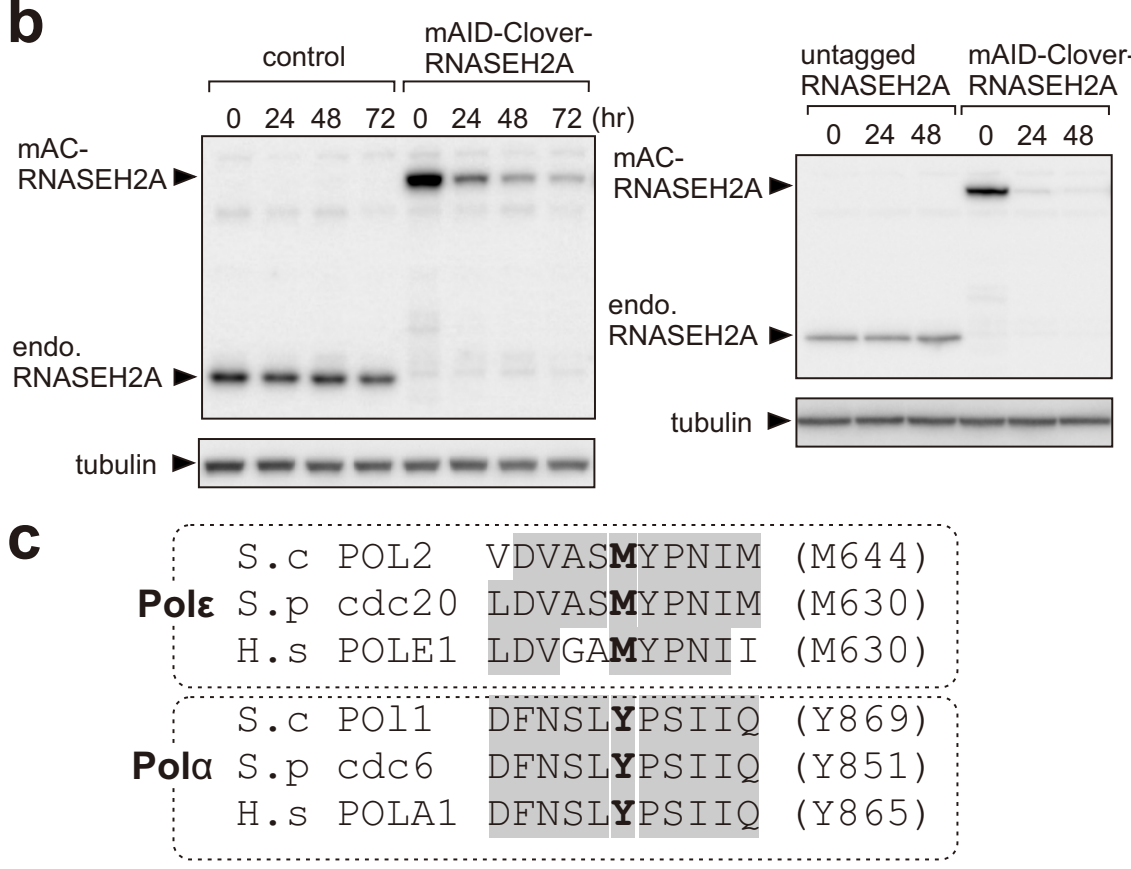

d
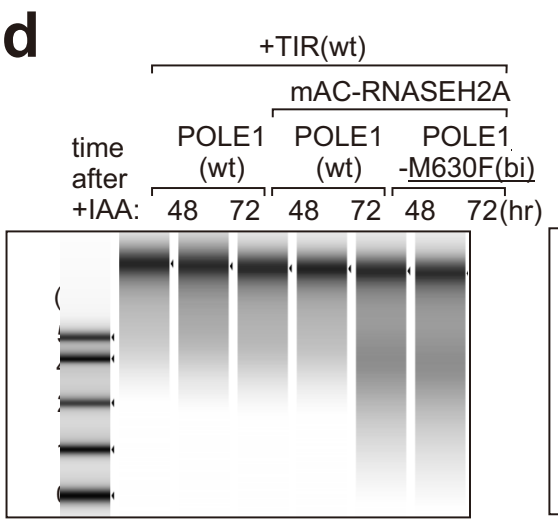

e

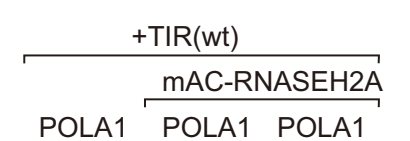

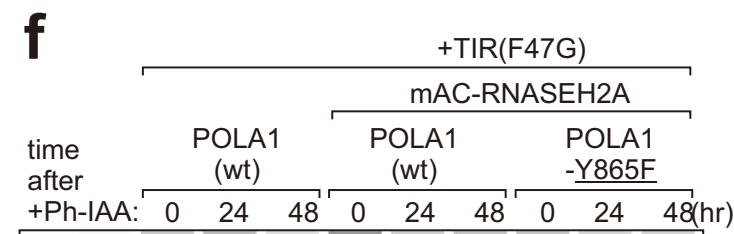

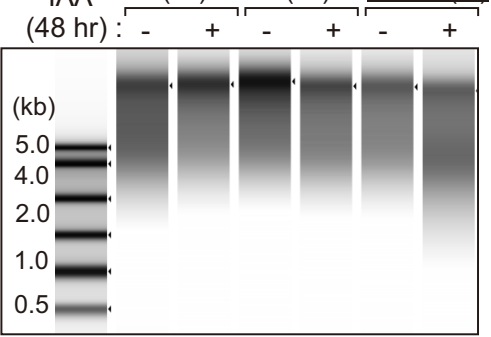

Fig. 1 | Ribonucleotide incorporation into DNA in POLE1-M630F and POLA1-Y865F cells.

a. Schematic representation of Pu-seq. Top: ribonucleotides $(R)$ are incorporated by the mutated DNA polymerase. Middle: in the absence of RNase H2-dependent RER, rNMPs remain in the DNA. Bottom: the sugar backbone of DNA strand is cleaved at sites of rNMP incorporation by alkali. Small ssDNA fragments are collected and subjected to library preparation and sequencing. $\mathbf{b}$. Left: auxin-induced degradation of RNASEH2A following addition of indole-3-acetic acid (IAA) to cells expressing wild type O. sativa TIR1 (AID system). Right: 5-Ph-IAA-induced degradation of RNASH2A in cells expressing O. sativa TIR1(F74G) (AID2 system). c. Conservation of targeted amino acid residues of DNA Pole and Pola to induce rNMP incorporation. d,e,f. Extracted genomic DNA from the indicated cell lines after the initiation of RNASEH2A degradation treated with alkali to cleave at incorporated rNMPs and analysed by electrophoresis. 
a

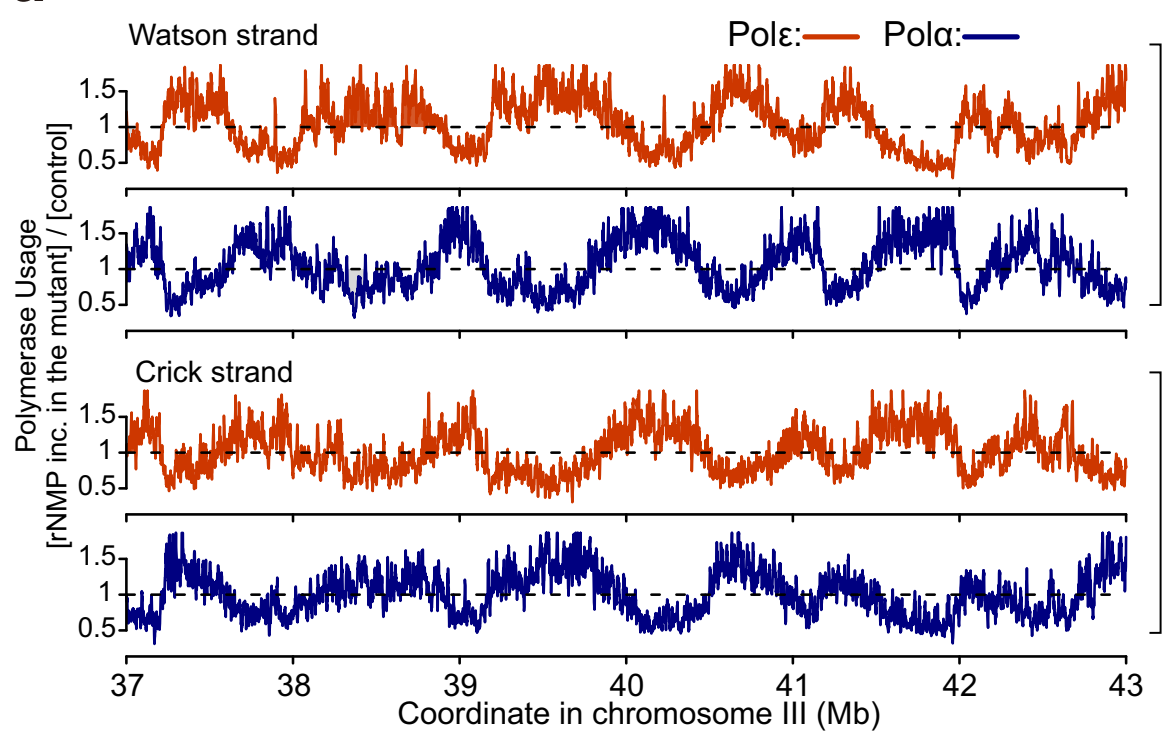

C
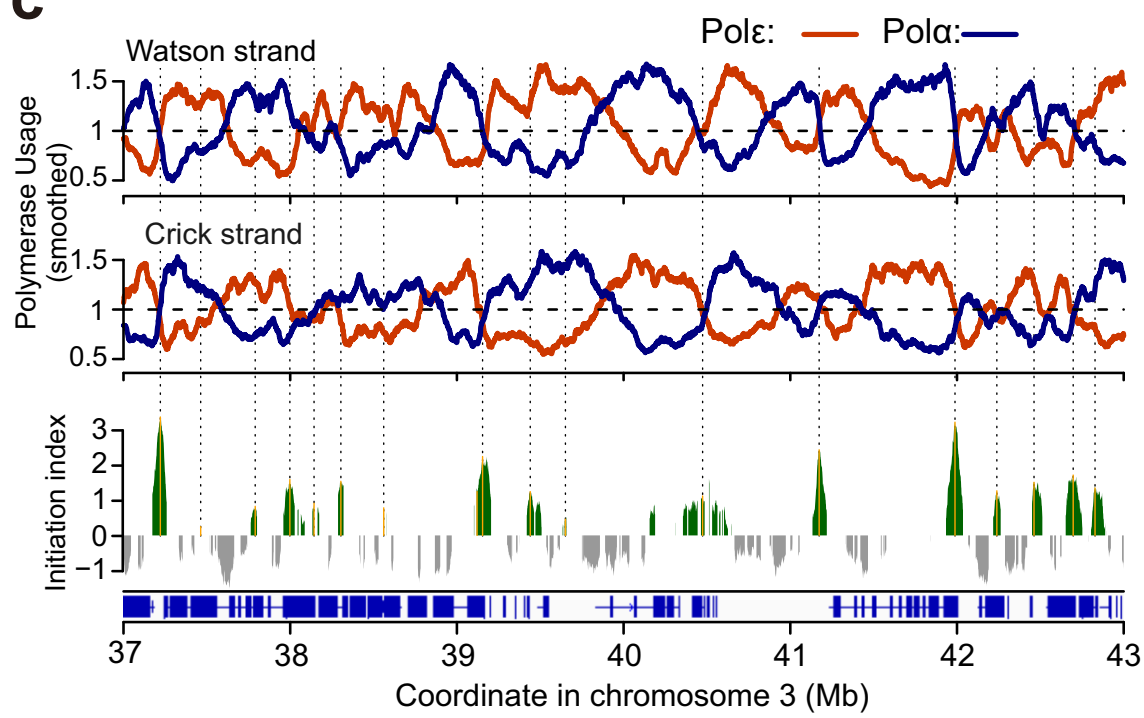

b
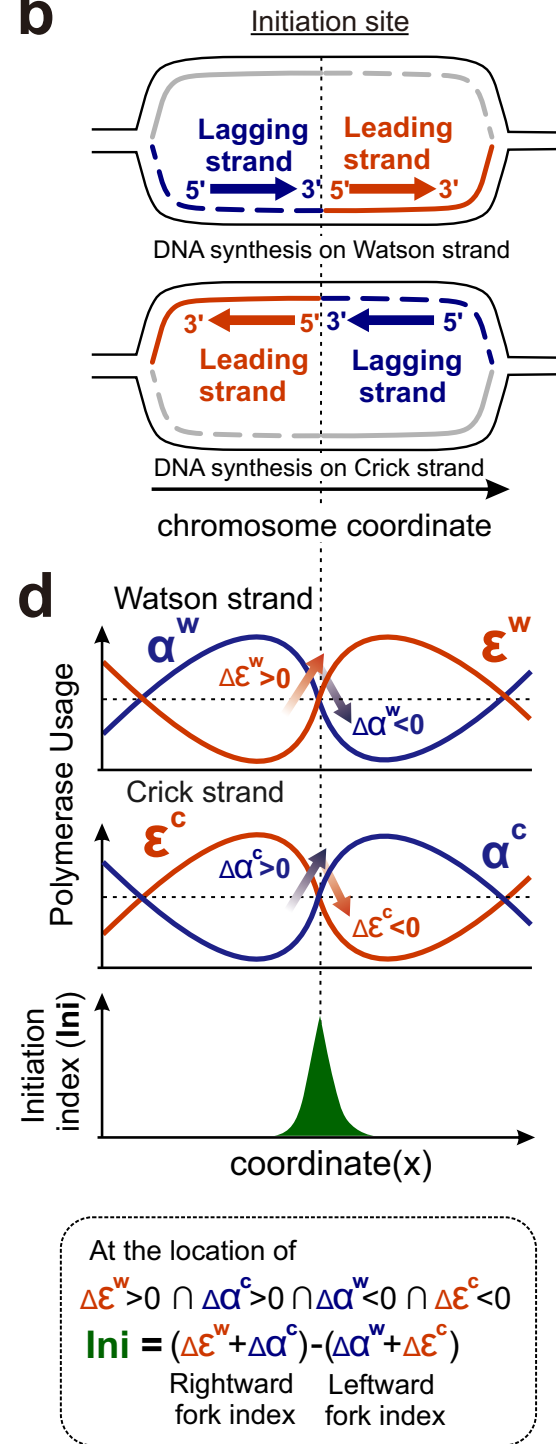

Fig. 2 | Polymerase usage and replication initiation across the human genome.

a. Profiles of the relative reads for Pole and Pola mutants on the Watson and Crick strand for each $1 \mathrm{~kb}$ bin. Orange: Polk (POLE1-M630F). Blue: Pola (POLA1-Y865F). A representative region of chromosome 3 is shown. Data were smoothed with a moving average $(m=3$; see materials and methods). $\mathbf{b}$. Schematic representation of predicted Pole and Pola profiles at a site of replication initiation. Orange: leading strand. Blue: lagging strand. c. Top: smoothed data from panel a (moving average, $m=30$ ) provides a map of polymerase usage. Bottom: plot of the calculated initiation index. Positive values (green) represent increased initiation activity. Negative values (grey) represent increased converging fork termination. $\mathbf{d}$. Definition of the initiation index (see materials and methods for further details). ' $\Delta$ ' indicates the differential between neighbouring bins, e.g. at location $x, \Delta \varepsilon(x)$ is defined as $\varepsilon(x+1)-\varepsilon(x)$. 
bioRxiv preprint doi: https://doi.org/10.1101/2021.11.14.468503; this version posted December 3, 2021. The copyright holder for this Fig. 3

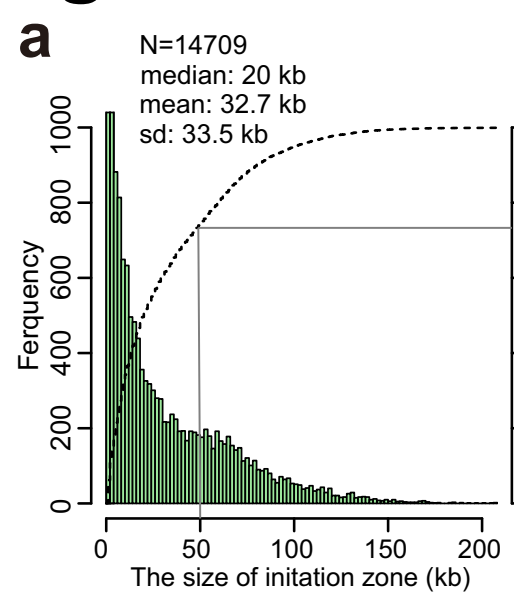

b

c
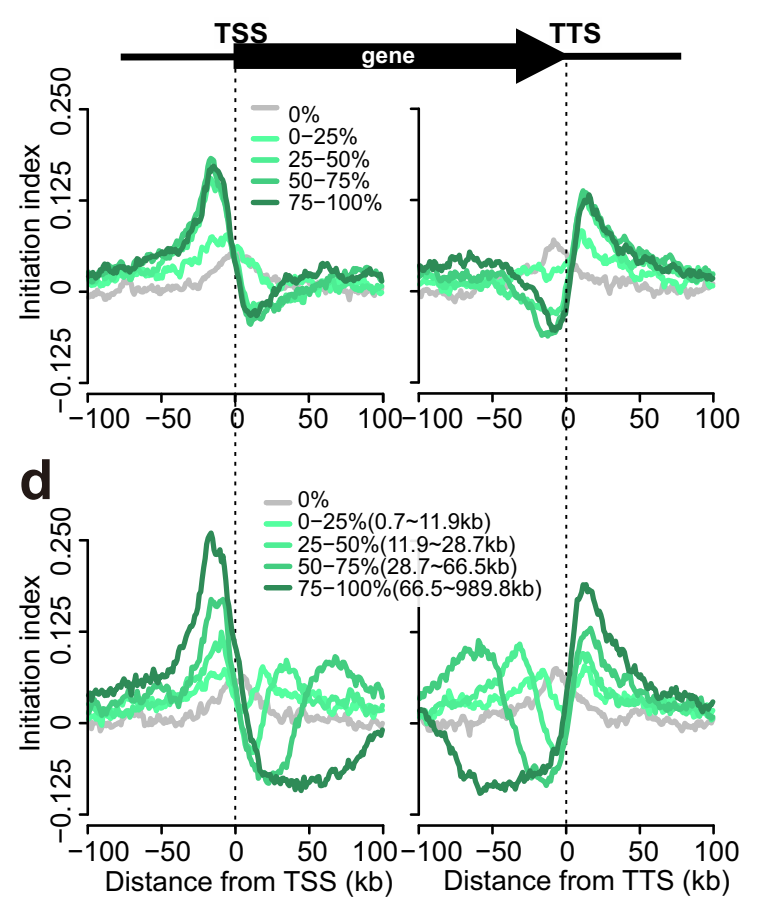

g
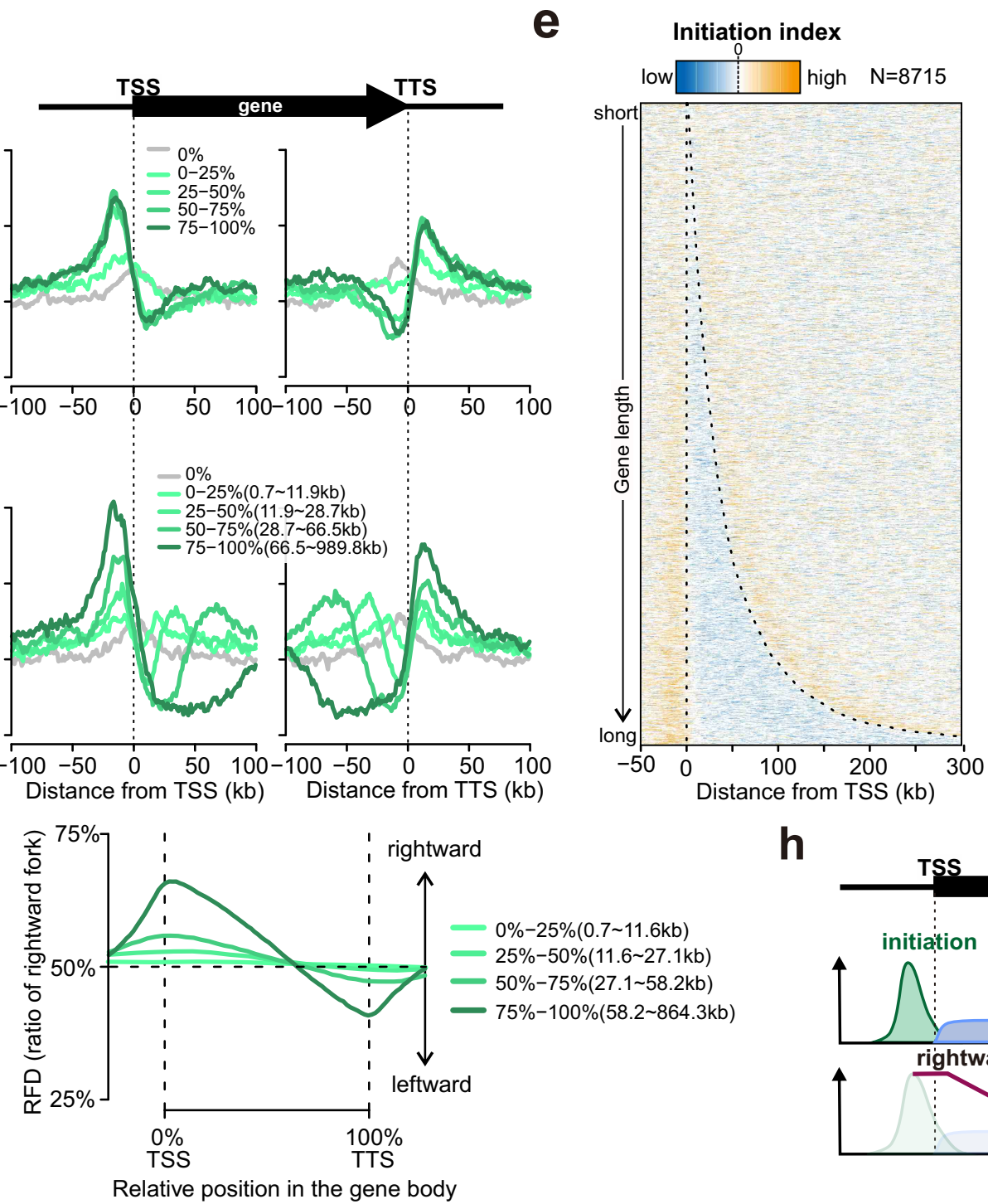

mosome $3(\mathrm{Mb})$

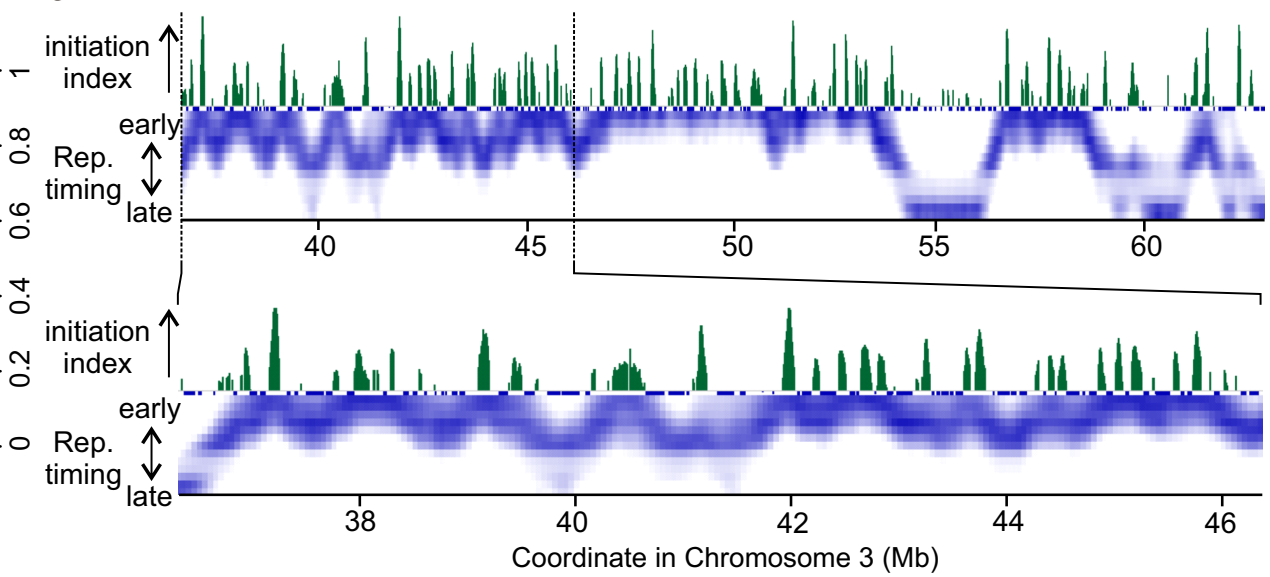

$\mathbf{e}$

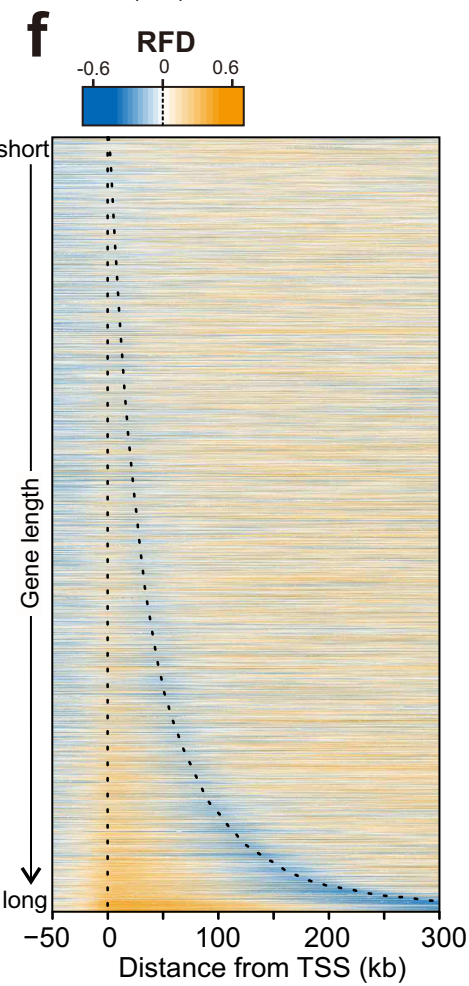

h

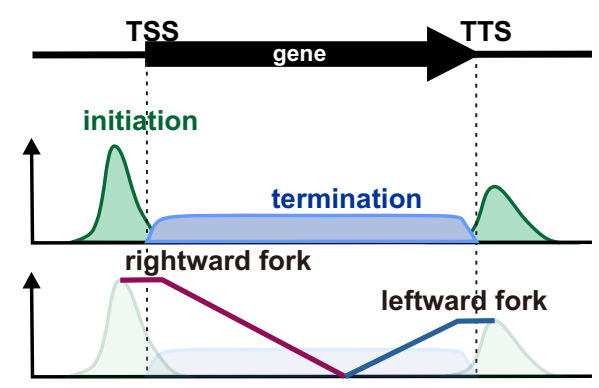

Fig. 3 | Genomic distribution of initiation sites.

a. Distribution of initiation zone widths. b. High-resolution replication timing data and initiation index plotted for a representative region of chromosome 3. The profile of replication timing for HCT116 cells is from Zao et al $(2020)^{21}$. c,d. Average initiation index $+/-100 \mathrm{~kb}$ around annotated TSS and TTS in the human genome. Initiation index data are categorised by transcriptional activity (c) or gene length (d). For the gene length analysis only the $50 \%$ most transcriptionally active genes were included. e. Heat map representation of data in panel d sorted by gene length. Broken lines indicate the position of TSS and TTS. f. Equivalent heat map representation of RFD aligned at TSS and TTS. g. Average RFD for the relative positions from TSS to TTS for all genes scaled to the same arbitrary length. RFD values from Supplementary Figure 2 were converted to rightward fork proportion. $\mathbf{h}$. Schematic representation of initiation and termination of replication forks as well as fork directionality around a representative gene. 
a

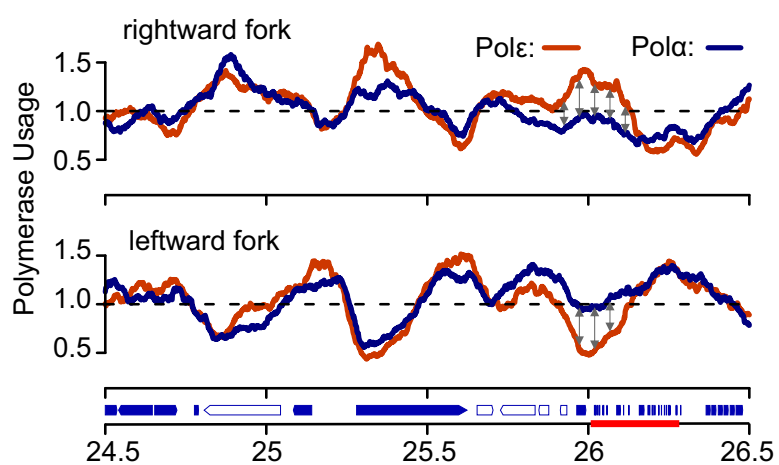

C

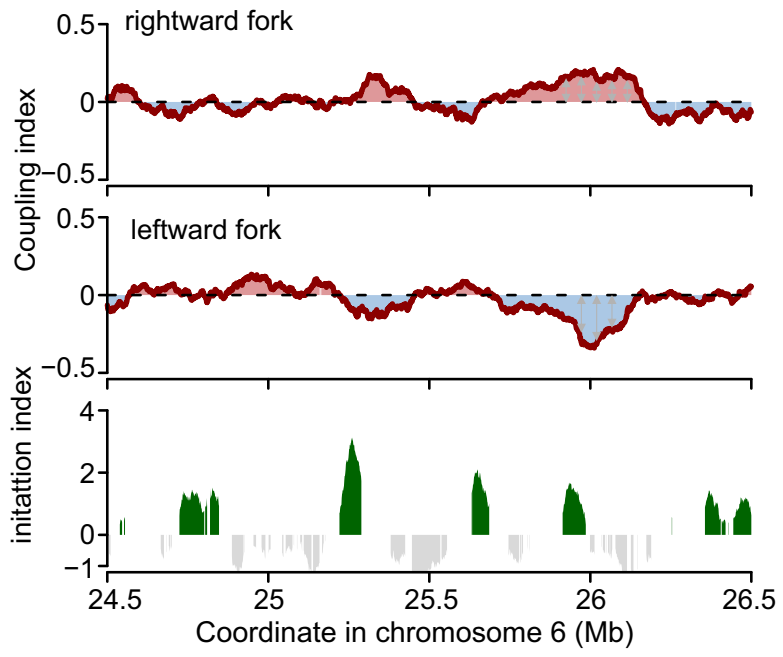

b

Coupling Index:

$\mathbf{C I}=\frac{[\text { leading; } \varepsilon]-[\text { lagging; } \alpha]}{[\text { leading; } \varepsilon]+[\text { lagging; } \alpha]}$
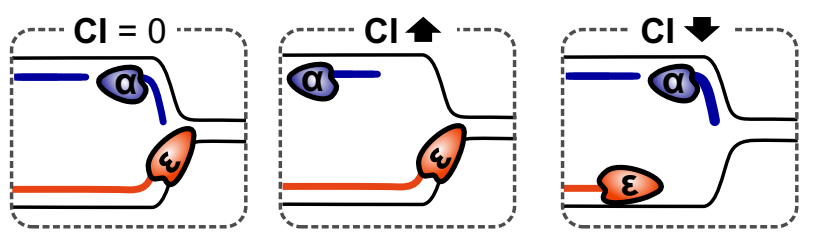

d

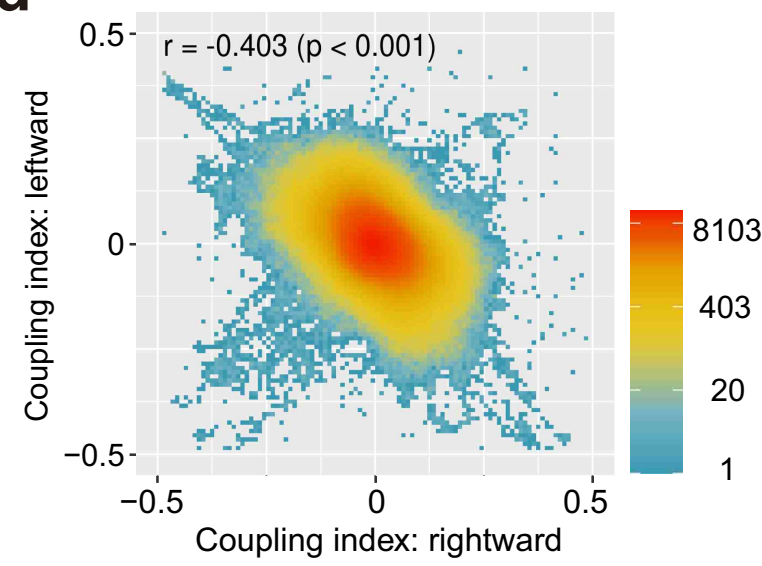

e

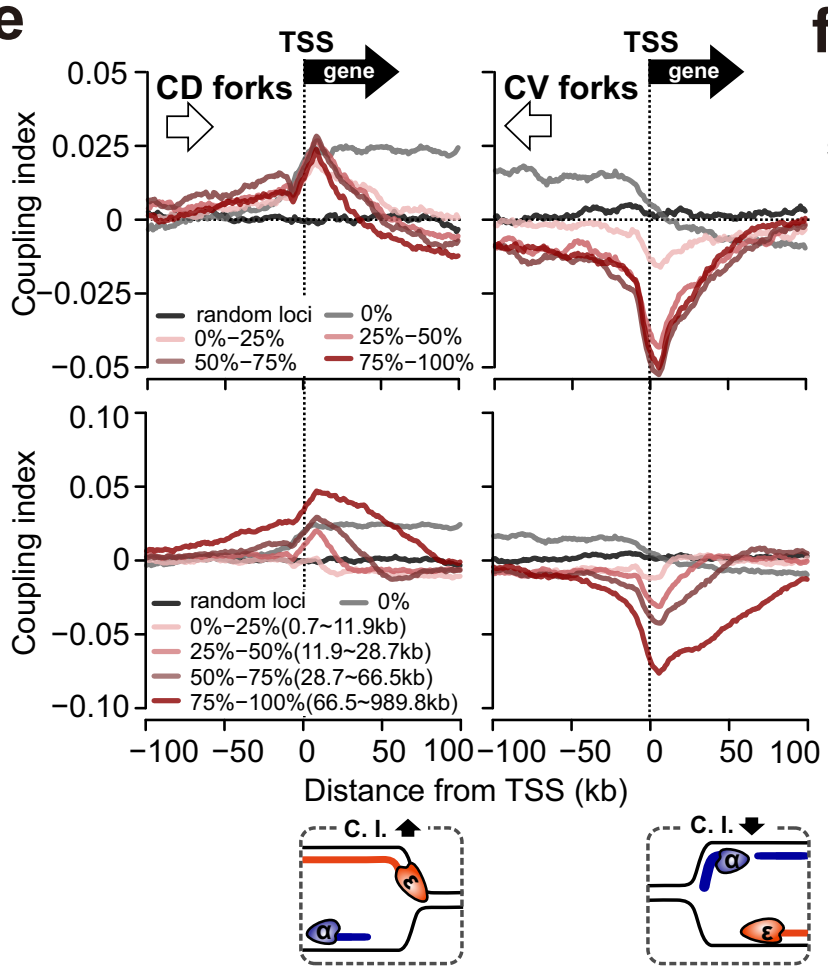

f

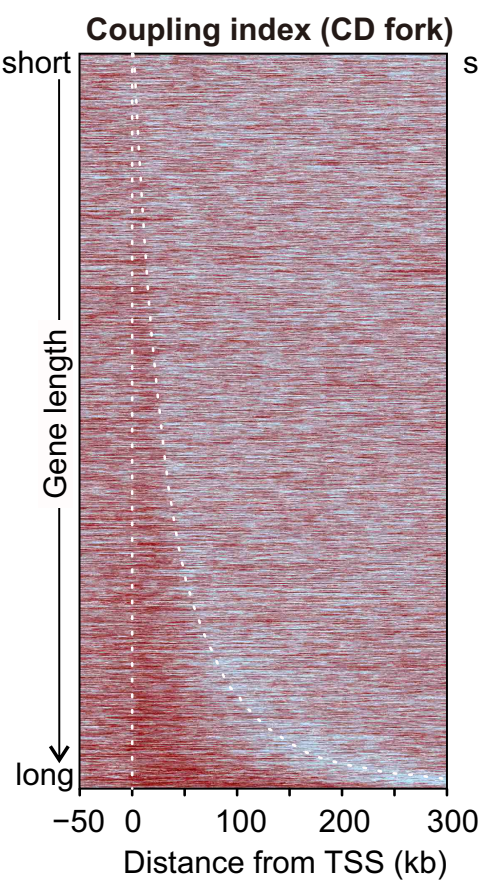

\begin{tabular}{c:cc}
-0.04 & 0 & -0.04 \\
\hline & & high $\mathrm{N}=8715$ \\
\hline & &
\end{tabular}

Coupling index (CV fork)
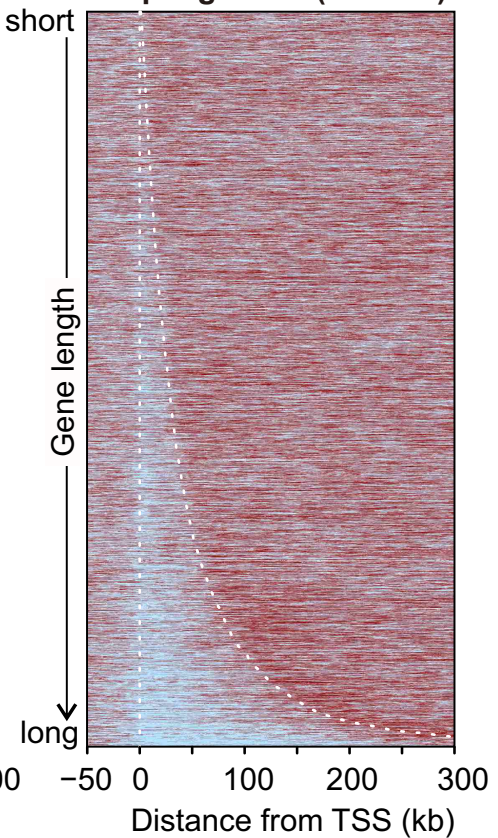

Fig. 5 | The uncoupling of leading and lagging strand DNA synthesis.

a. Profiles of polymerase usage for a region of chromosome 6 . Arrows on the vertical axis indicate active genes (blue-filled) and inactive genes (unfilled). The red line on the vertical axis indicates a cluster of histone-encoding genes. b. Definition of coupling index ( $\mathrm{Cl}$, for details see materials and methods). c. Top: $\mathrm{Cl}$ of rightward moving forks. Middle: $\mathrm{Cl}$ of leftward moving forks. Bottom: the initiation index of the same region for comparison. d. Correlation of $\mathrm{Cls}$ of rightward and leftward forks. e. Averaged coupling index $+/-$ $100 \mathrm{~kb}$ around annotated TSS in the human genome for CV and CD forks categorised by transcriptional activities (top) or gene length (bottom). Only the $50 \%$ most transcriptionally active genes were included. f. Heat map representation of data in panel e. Data were sorted by gene length. Broken lines indicate the positions of TSS and TTS. 


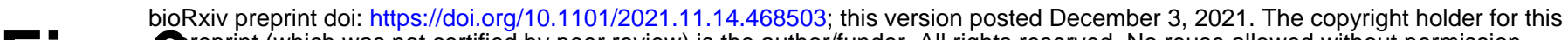
Fig. 6

a

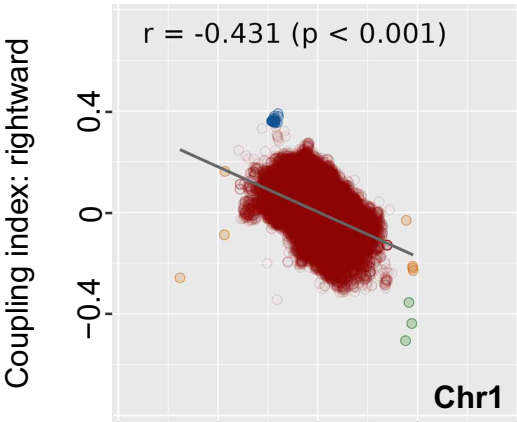

$\begin{array}{lll}-0.4 & 0 & 0 \\ 0 & 0 & 4\end{array}$

Coupling index: leftward

b

Chr3: left arm
rightward fork
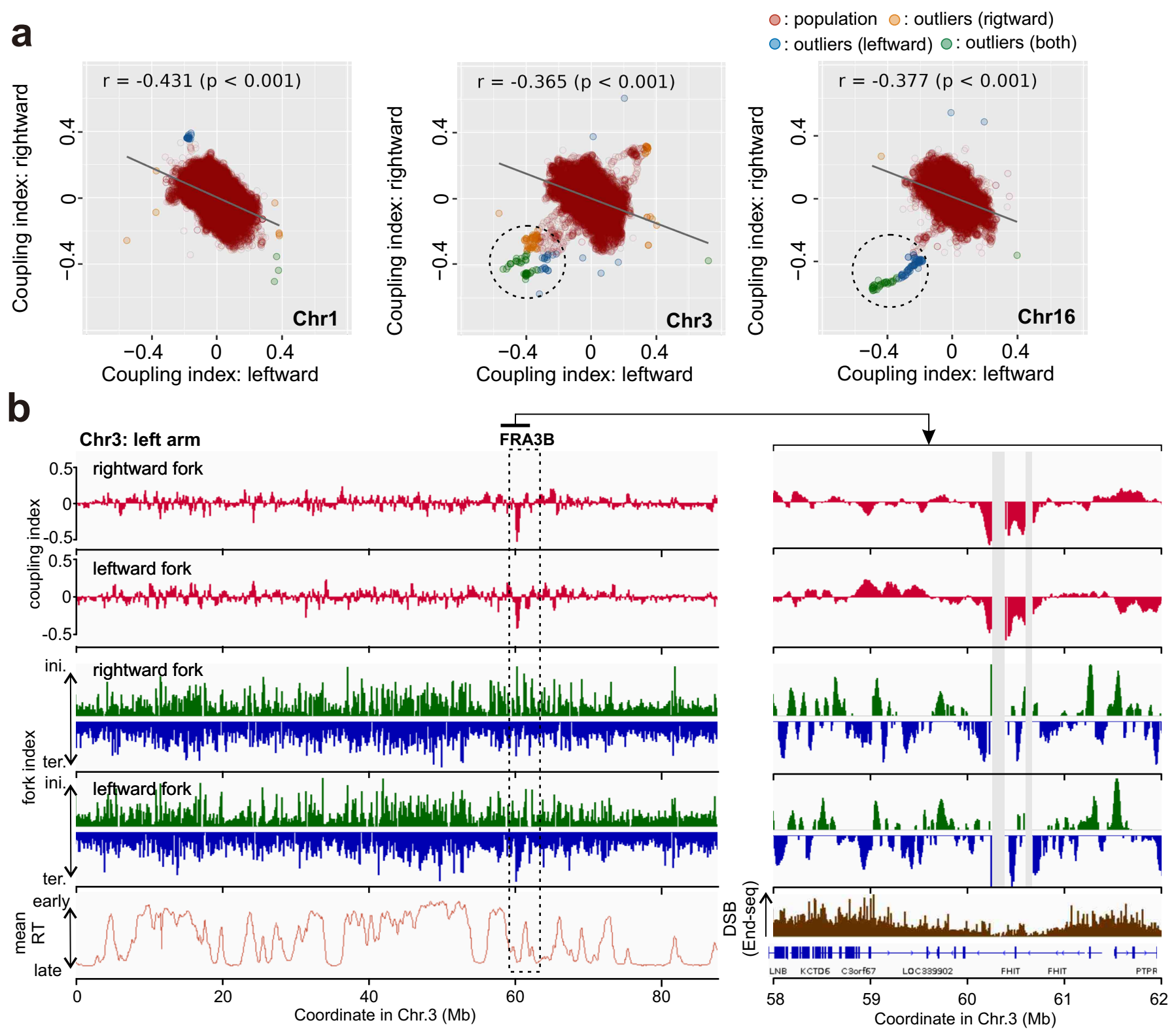

C

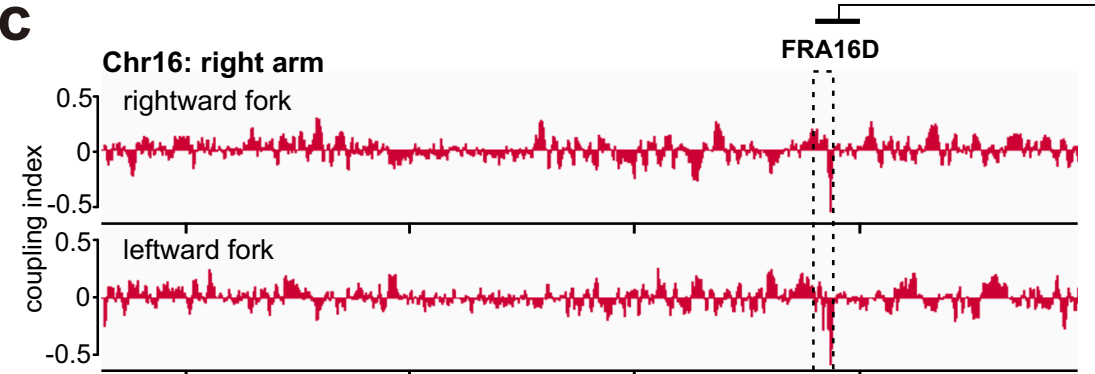

ini. rightward fork
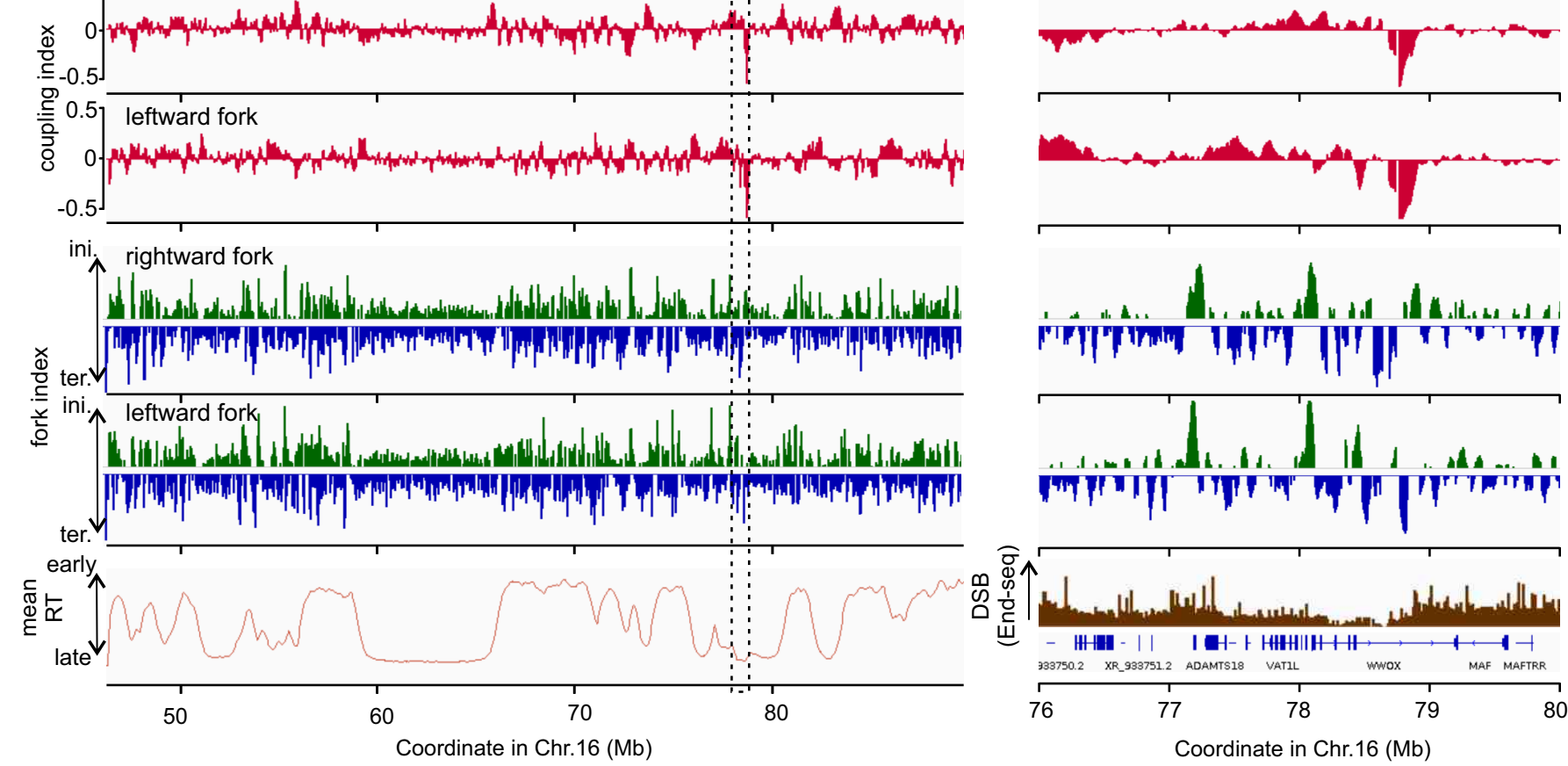

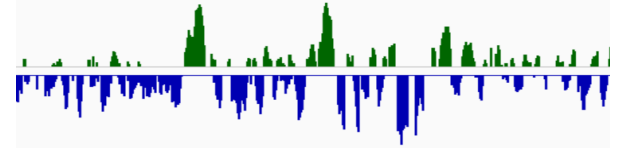

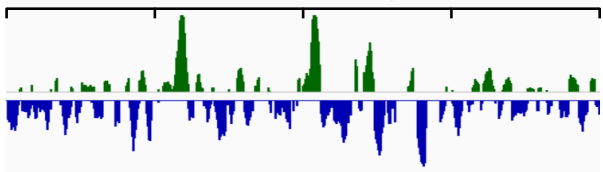

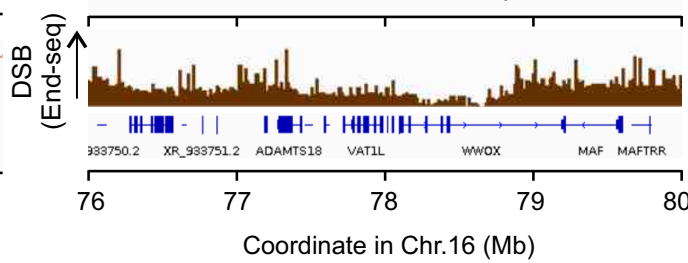

(The legend is on the next page.) 
Fig. 6 | Polymerase uncoupling at common fragile sites FR3B and FRA16D.

a. Correlation of coupling index of rightward and leftward moving forks presented by chromosome. Chromosomes 1, 3 and 16 are shown. The circles highlight data points dispersed from the bulk of population. These outliner data points were identified by Smirnov-Grubbs test. b. Top: profiles of coupling index for rightward and leftward forks. Middle: fork index profile. Bottom: mean replication timing (mean RT) or DSB data for the FRA3B region on chromosome 3. c. The equivalent data for the FRA16D region of chromosome 16. Replication timing data for HCT116 cells are derived from Zao et al (2020) $)^{21}$. Data of DSBs from End-seq are derived from Tubbs et al $(2018)^{29}$. 


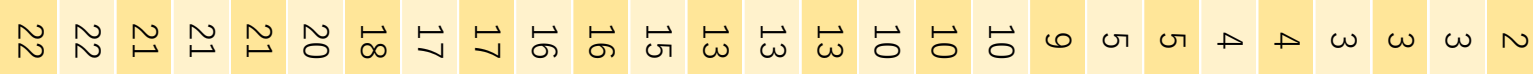

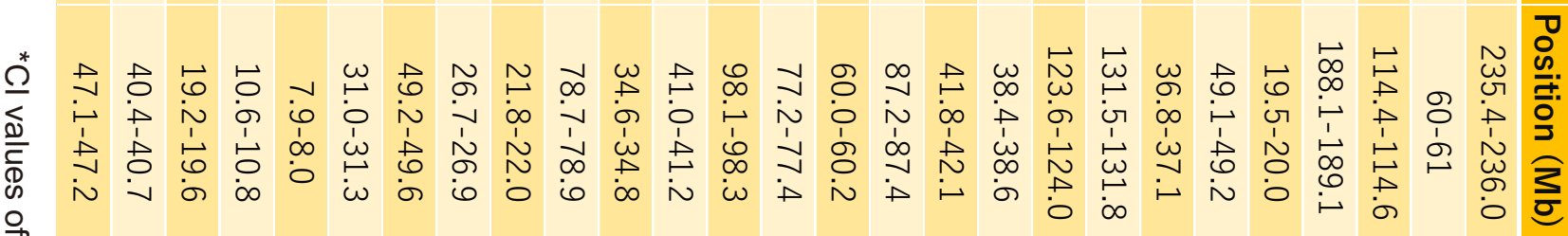

棉

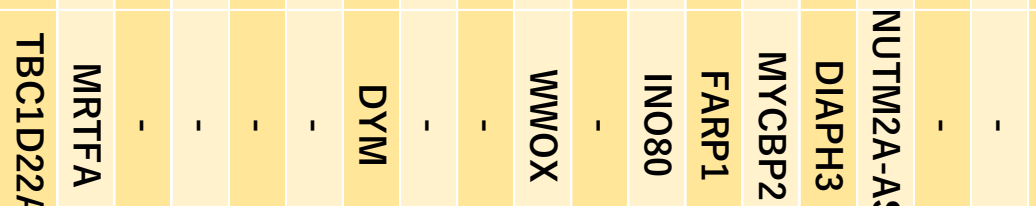

N

$\underset{\omega}{\omega} \stackrel{N}{\sigma}$

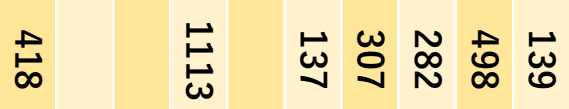

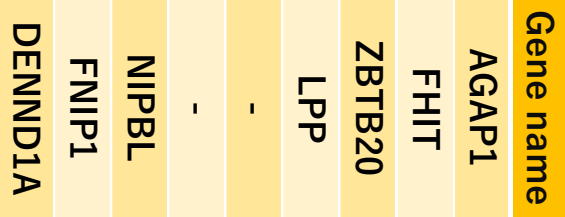

$\underline{\underline{O}}$

웃

$\underset{\mathbb{1}}{\mathbb{1}}$

$\frac{\text { D }}{\stackrel{0}{\frac{1}{D}}}$

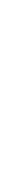

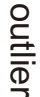

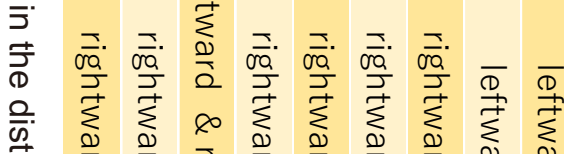

$\frac{\mathrm{O}}{\mathrm{D}}$

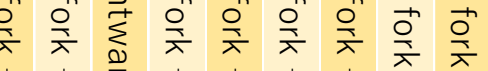

\section{$\stackrel{\uparrow}{\stackrel{\frac{D}{D}}{+}}$}

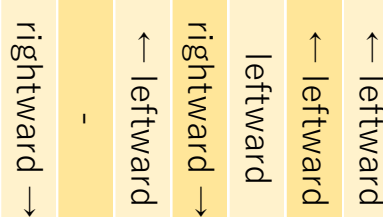

킹 ज्ञ

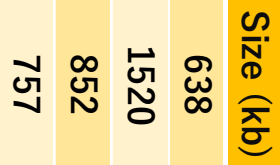

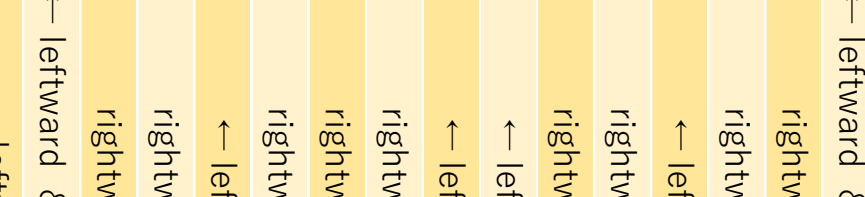

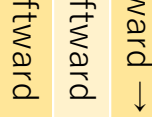

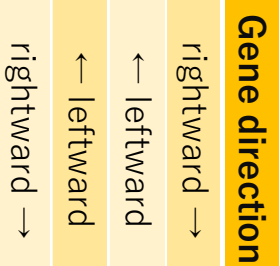

8 
Table 2, Resource table

\begin{tabular}{|c|c|c|}
\hline REAGENT & SOURCE & IDNTFIER \\
\hline \multicolumn{3}{|l|}{ Cell lines } \\
\hline HCT116 +TetOsTIR(bi) & Natsume et al. 2016 & $\begin{array}{l}\text { RIKEN NBRP } \\
\text { RCB4662 }\end{array}$ \\
\hline HCT116 Tet-OsTIR(bi)::AAVS1(bi) RNASEH2A-mAID-Clover::hygR(bi) & This study & \\
\hline $\begin{array}{l}\text { HCT116 Tet-OsTIR(bi)::AAVS1(bi) RNASEH2A-mAID-Clover::hygR(bi) } \\
\text { POLE1-L630F(bi) }\end{array}$ & This study & \\
\hline HCT116 CMV-OsTIR(F74G)::AAVS1(bi) & $\begin{array}{l}\text { Yesbolatova et al. } \\
2020\end{array}$ & \\
\hline HCT116 CMV-OsTIR(F74G)::AAVS1(bi) RNASEH2A-mAID-Clover(bi) & This study & \\
\hline $\begin{array}{l}\text { HCT116 CMV-OsTIR(F74G)::AAVS1(bi) RNASEH2A-mAID-Clover(bi) } \\
\text { POLA1-Y865F(bi) }\end{array}$ & This study & \\
\hline \multicolumn{3}{|l|}{ Oligo DNA } \\
\hline $\begin{array}{l}\text { GATGAGATTAAGAGCAAGCTTGCCTCCCTGAAGGACGTTCCCAG } \\
\text { CCGCATCGAGTGTCCACTCATCTACCACCTGGACGTGGGGGCCtT } \\
\text { cTACCCCAACATCATCCTGACCAACCGCCTGCAGGTGA }\end{array}$ & $\begin{array}{l}\text { IDT Alt-R }{ }^{\text {TM }} \text { HDR Donor Oligo, } \\
\text { custom production }\end{array}$ & $\begin{array}{l}\text { SSODN- } \\
\text { POLE1- } \\
\text { M630F }\end{array}$ \\
\hline $\begin{array}{l}\text { TTCTTTAGACTTTTTATGACGTGGCTTTTTAATTTCAGGTTTTTATGA } \\
\text { TAAGTTCATTTTGCTTCTGGACTTCAACAGTCTATtCCCTTCCATCAT } \\
\text { TCAGGAATTTAACATTTGTTTTACAACAGTA }\end{array}$ & $\begin{array}{l}\text { IDT Ultramer DNA Oligo, custom } \\
\text { production }\end{array}$ & $\begin{array}{l}\text { SSODN- } \\
\text { POLA1- } \\
\text { Y865F }\end{array}$ \\
\hline \multicolumn{3}{|l|}{ Oligo RNA } \\
\hline $\begin{array}{l}\text { IAITR1/CAGGAUGAUGUUGGGGUACAGUUUUAGAGCUAUGCU/AI } \\
\text { TR2/ }\end{array}$ & Alt-R CRISPR-Cas9 crRNA & $\begin{array}{l}\text { CrRNA- } \\
\text { POLE1- } \\
\text { M630F }\end{array}$ \\
\hline $\begin{array}{l}\text { IAITR1/UUAAAUUCCUGAAUGAUGGAGUUUUAGAGCUAUGCU/AI } \\
\text { TR2/ }\end{array}$ & Alt-R CRISPR-Cas9 crRNA & $\begin{array}{l}\text { CrRNA- } \\
\text { POLA1-Y865 }\end{array}$ \\
\hline \multicolumn{3}{|l|}{ Plasmid DNA } \\
\hline px330-RNaseH2A-C-terminal & This study & \\
\hline pBS-RNaseH2A -mAID-Clover-Hygro-donor & This study & \\
\hline
\end{tabular}


Replicate 1

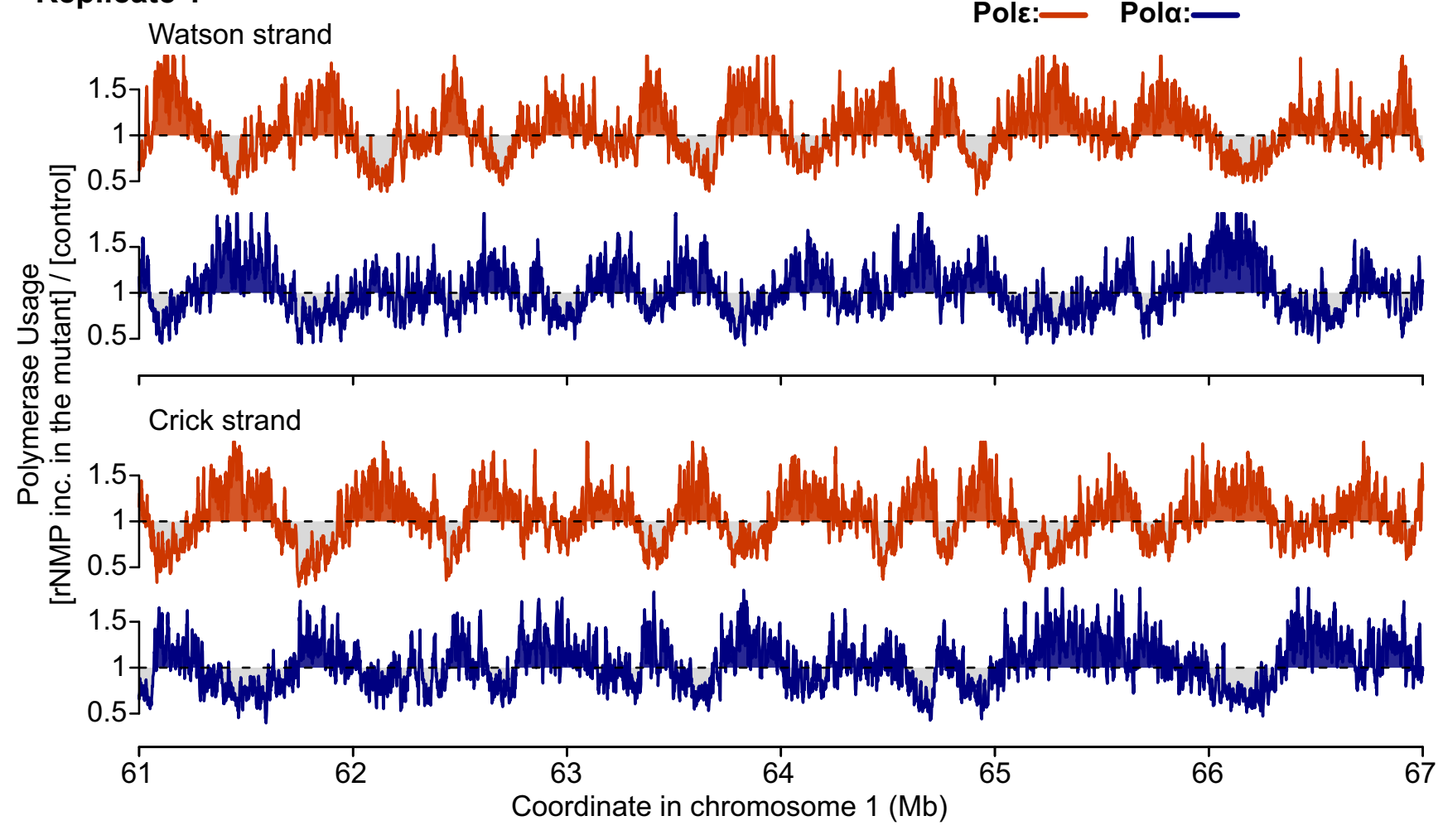

Replicate 2

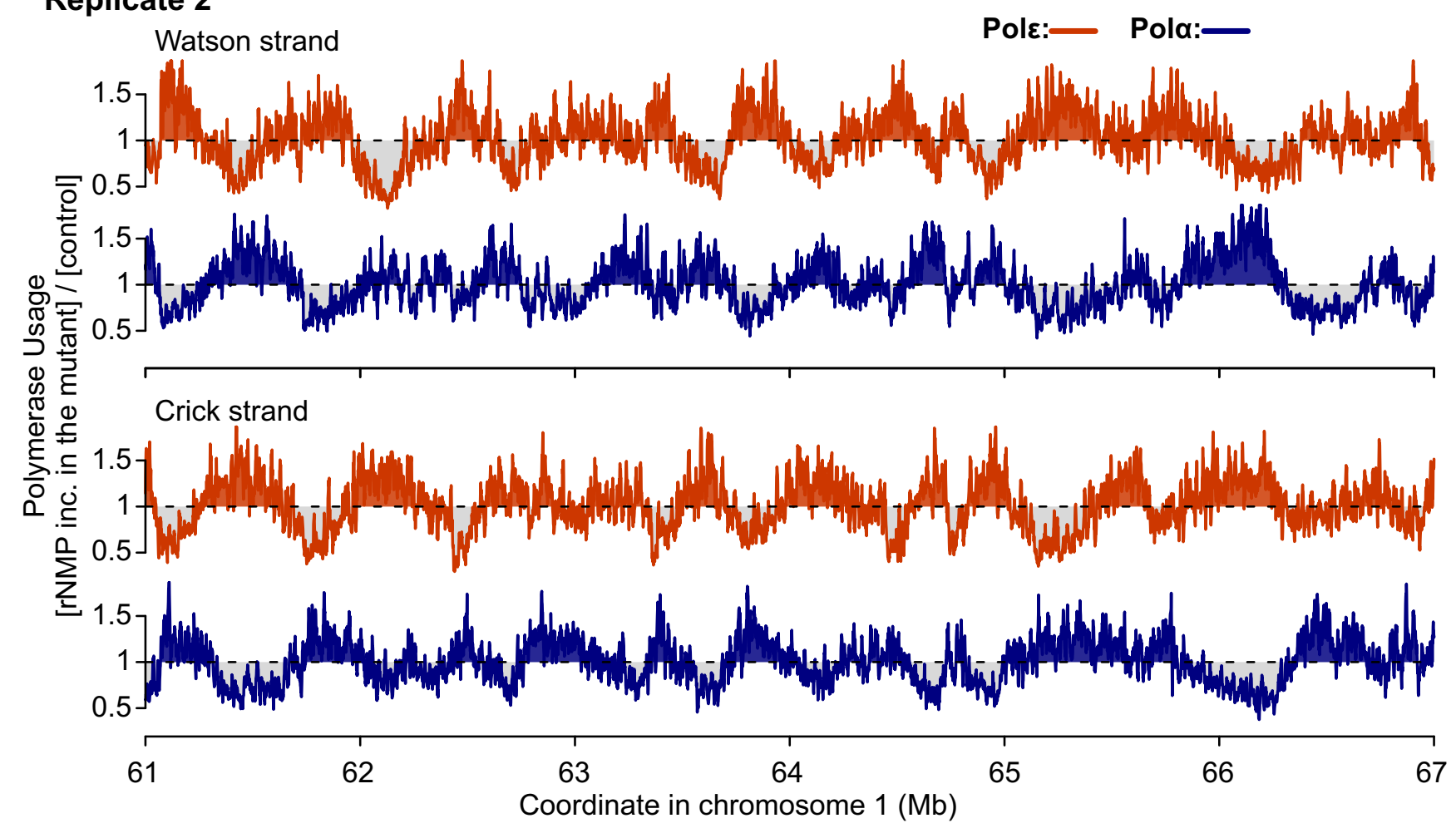

Supplementary fig. 1 | Polymerase usage of 2 experimental replicates across the human genome. For two independent experiments (Replicate 1 and Replicate 2) the profiles of the relative reads for Pol $\varepsilon$ and Pola mutants on the Watson and Crick strand for each $1 \mathrm{~kb}$ bin are shown. Data are normalised to Pol+. Orange: Polk (POLE1-M630F). Blue: Pola (POLA1-Y865F). A representative region of chromosome 1, a different location to that in Fig. 2, is shown. Data were smoothed with a moving average $(m=3$; see materials and methods). 


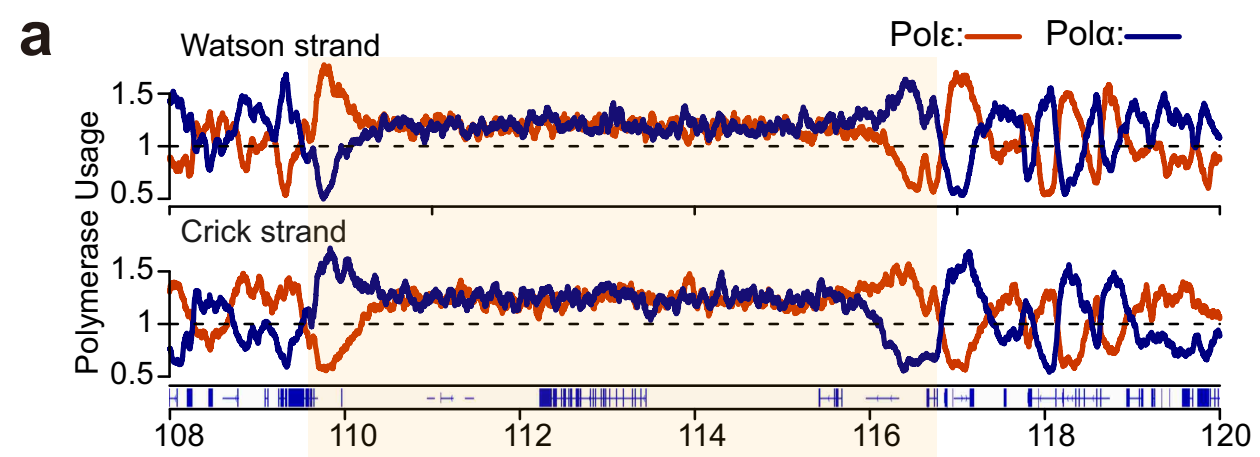

b
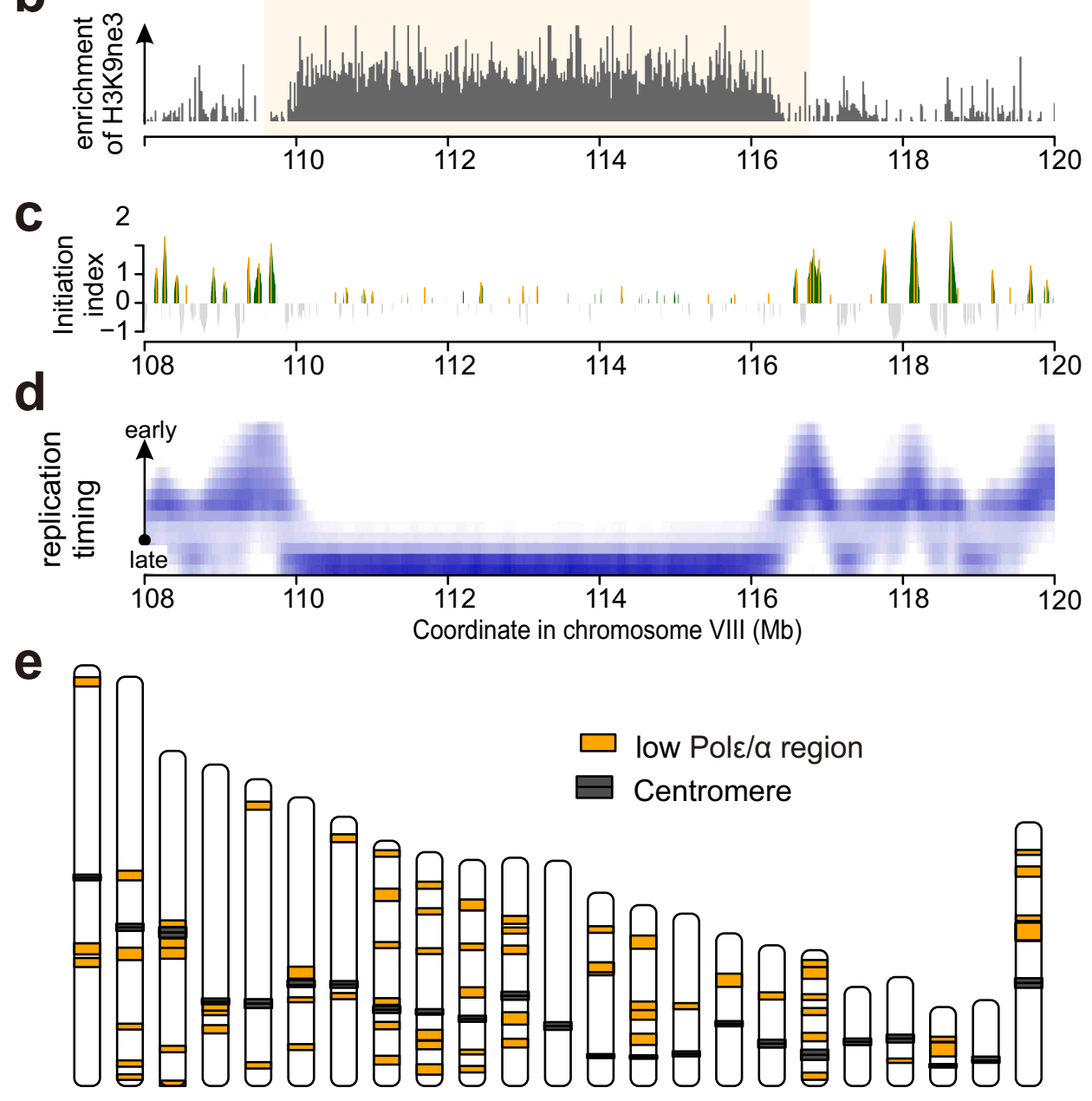

Supplementary fig. 2 | Polymerase usage and replication initiation around late replicating regions. a. Polymerase usage profiles of Pole (orange) and Pola (blue) plotted for a representative region showing an equal profile of polymerase usage. Light orange background represents such a 'low Polz/a region'. b. H3K9me3 enrichment at the same region. Data are taken from Lay et al (2015) ${ }^{49}$. H3K9me3 marks heterochromatin. c. Initiation index plotted for the same region. d. Replication timing in HCT116 cells. RT data are taken from Zao et al $(2020)^{21}$. e. Schematic of the genome-wide distribution of 'low Pole/a regions'. 
a Replication fork directionality (RFD):

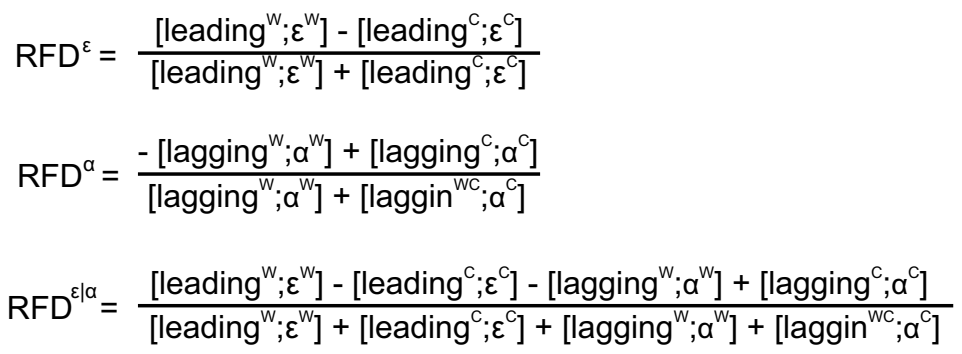
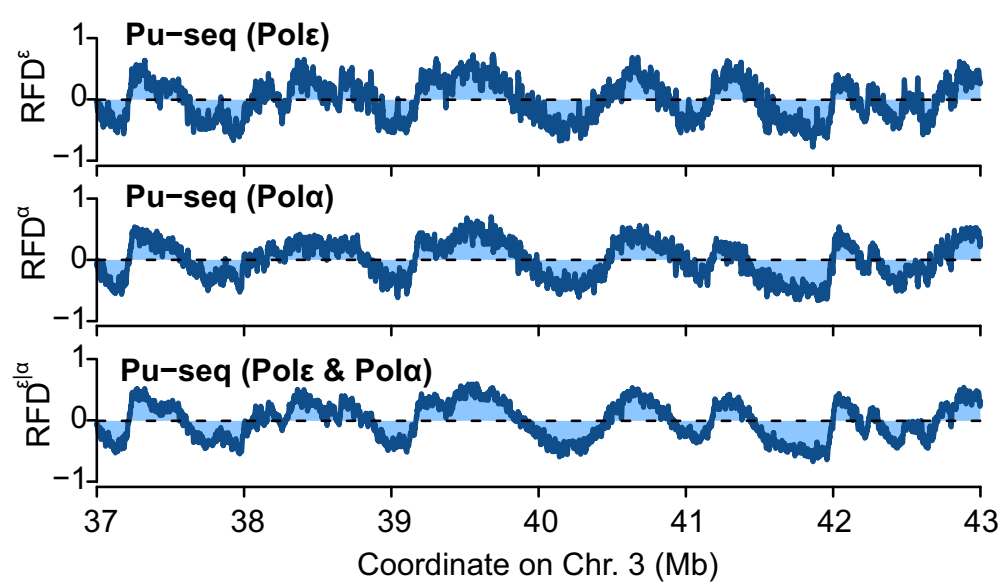

b

$\operatorname{RFD}^{\circ \mathrm{K}}=\frac{\left[\left[\text { Okazaki } \mathrm{fr}^{\mathrm{c}}\right]-\left[\text { Okazaki } \mathrm{fr}^{\mathrm{W}}\right]\right.}{\left[\text { Okazaki } \mathrm{fr}^{\mathrm{W}}\right]+\left[\mathrm{Okazaki}_{\mathrm{fr}}^{\mathrm{c}}\right]}$
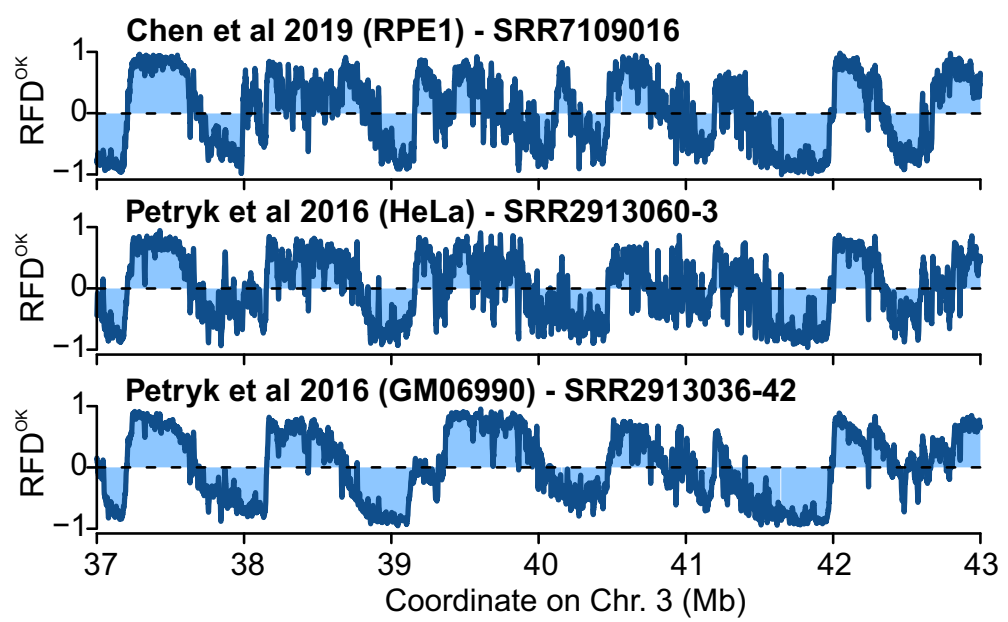

C

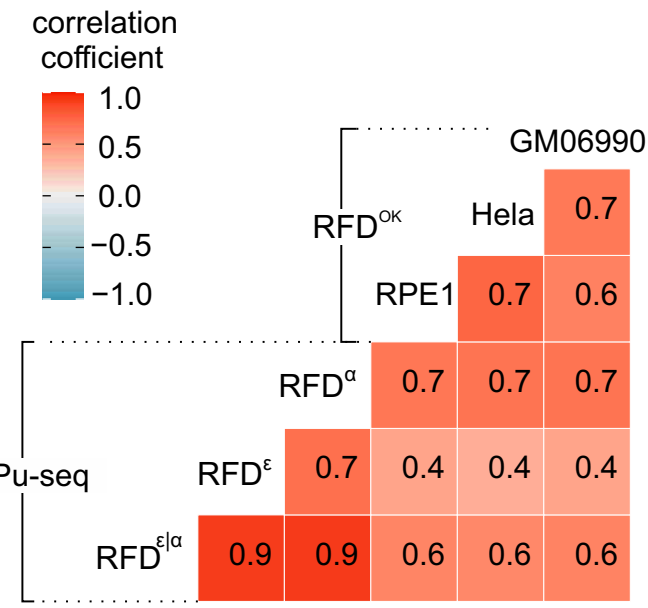

Supplementary fig. 3 | Replication fork directionality (RFD) from Pu-seq and OK-seq.

a. RFDs are calculated from Pole $\left(\operatorname{RFD}^{\varepsilon}\right)$ Pola $\left(\operatorname{RFD}^{\alpha}\right)$ or both data sets $\left(\operatorname{RFD}^{\varepsilon \mid \alpha}\right)$ and plotted for a representative section of chromosome 3. b. RFDs are calculated from three published OK-seq datasets and plotted for the same representative region. c. Correlation among RFDs from Pu-seq and OK-seq. 
a

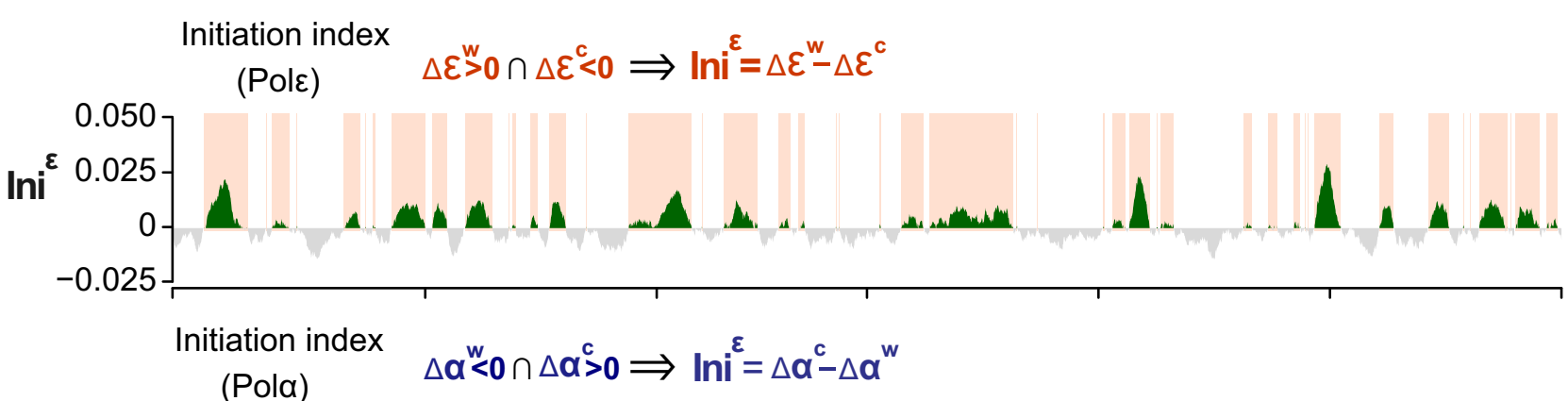

b (Pola)

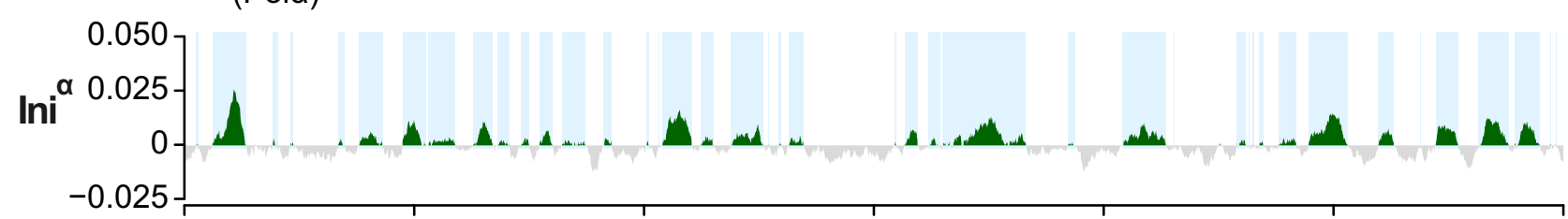

C

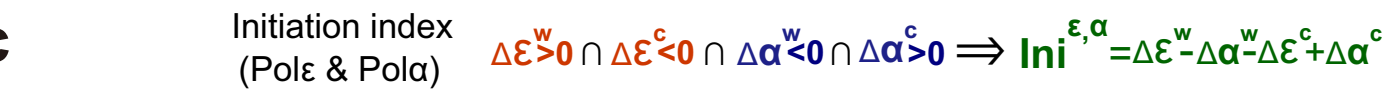

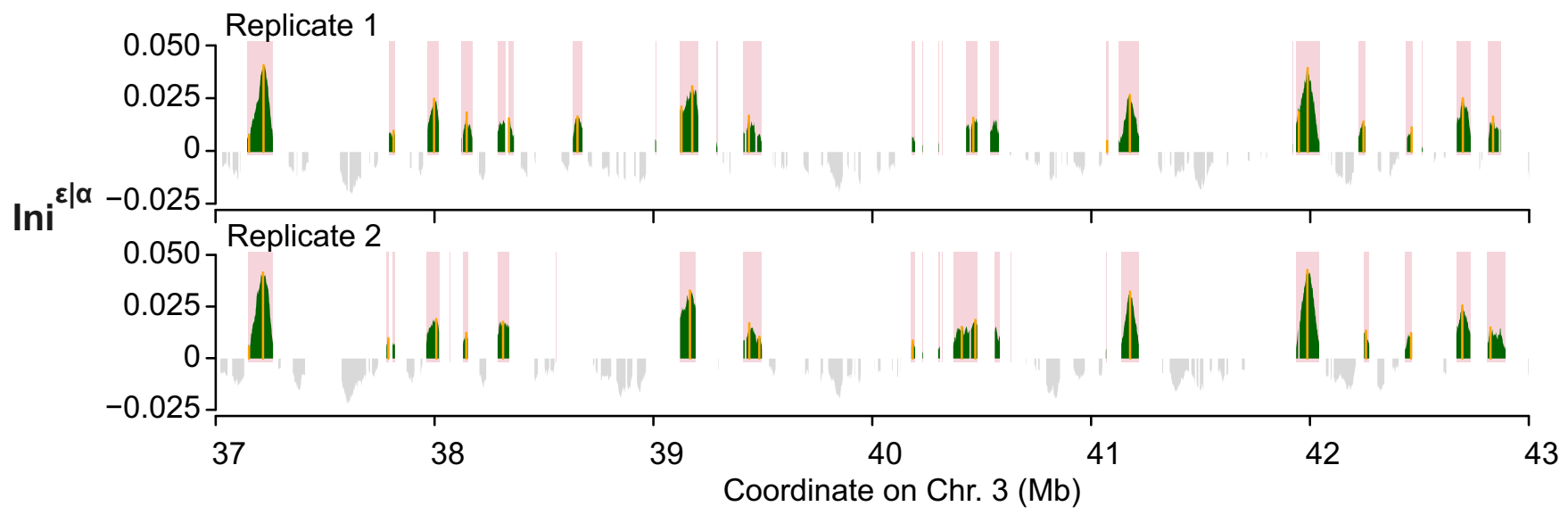

d

e
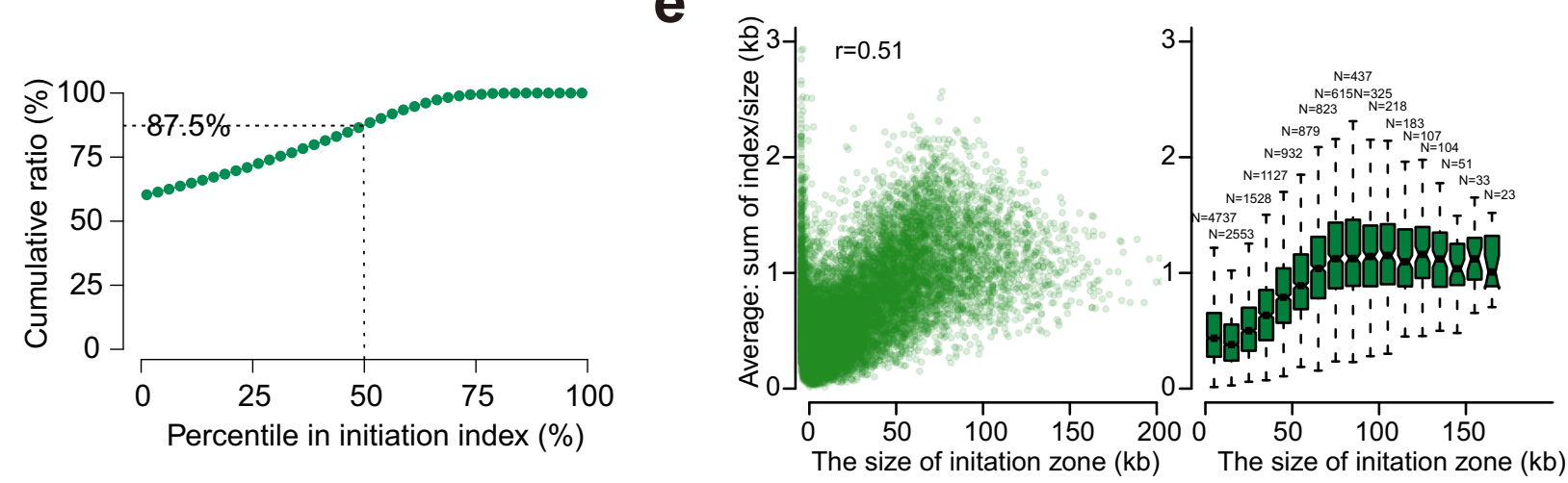

Supplementary fig. 4 | Genome-wide initiation index identifies replication initiation zones.

a. Initiation index calculated from Pole and plotted for a representative region of chromosome 3. Replication initiation sites are defined as the positive zones of initiation index and are marked with a coloured background. b. Initiation index calculated from Pola data and plotted as in panel a. c. Initiation index calculated from combined Pol $\varepsilon$ and Pola data and plotted as in panel a. The two independent experimental replicates are shown. Yellow vertical lines indicate computationally identified location of peaks within the zone. d. Cumulative ratio of replication initiation sites which locate at the concordant regions between the two experimental replicates. $\mathbf{e}$. The relationship of initiation index with the width of initiation zones. 


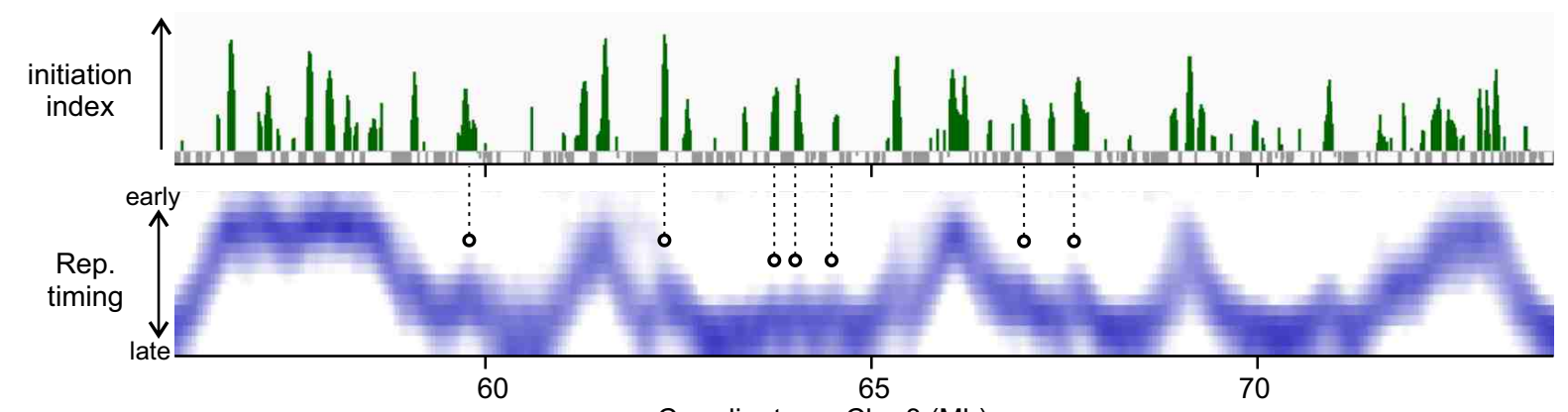

Coordinate on Chr. $3(\mathrm{Mb})$

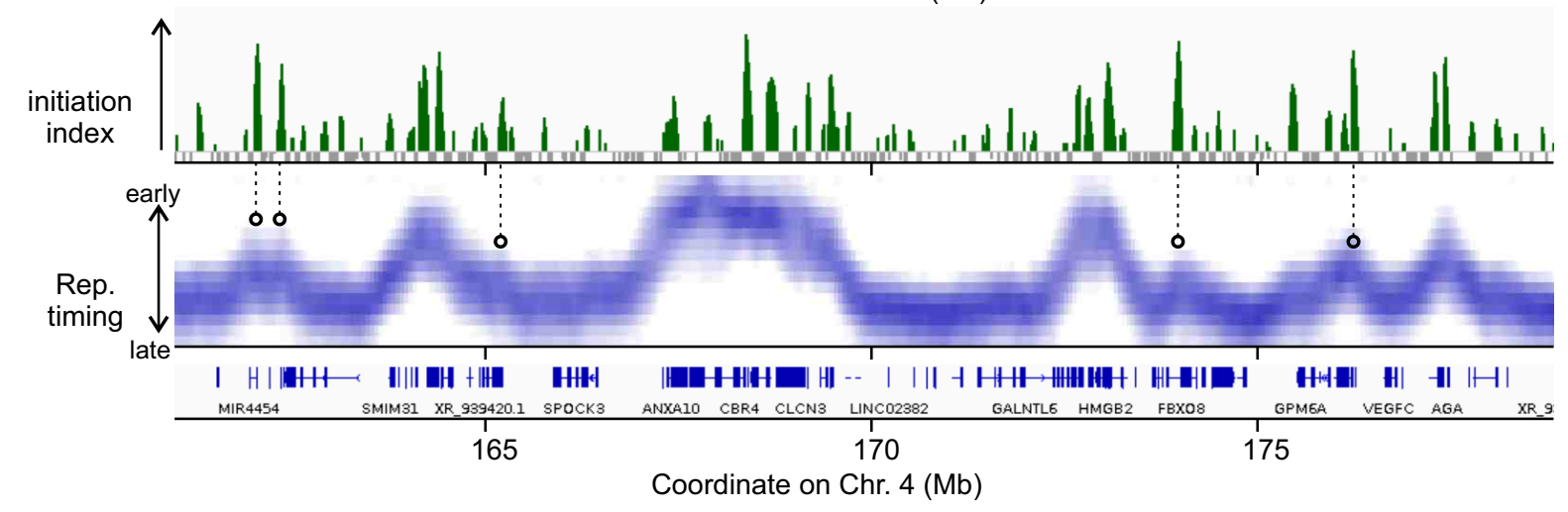

Supplementary fig. 5 | Genome-wide initiation index compared to replication timing.

High-resolution replication timing data and initiation index plotted for a representative region of chromosome 3 (top) and chromosome 4 (bottom). The profile of high resolution replication timing for HCT116 cells are from Zao et al $(2020)^{21}$. 
a

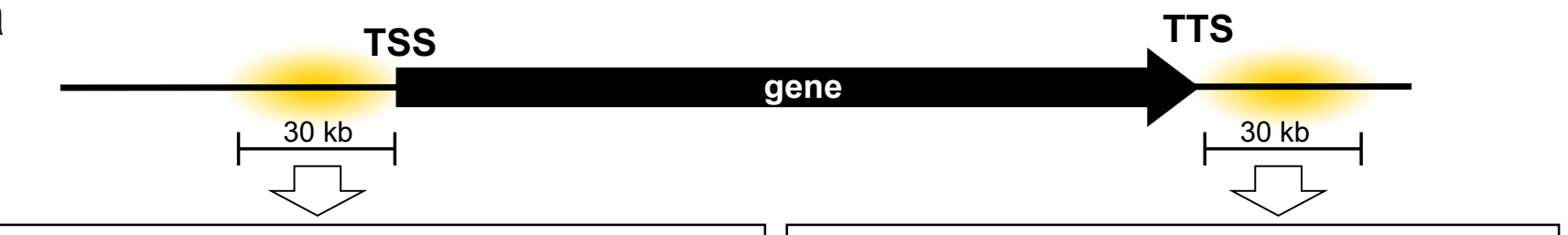

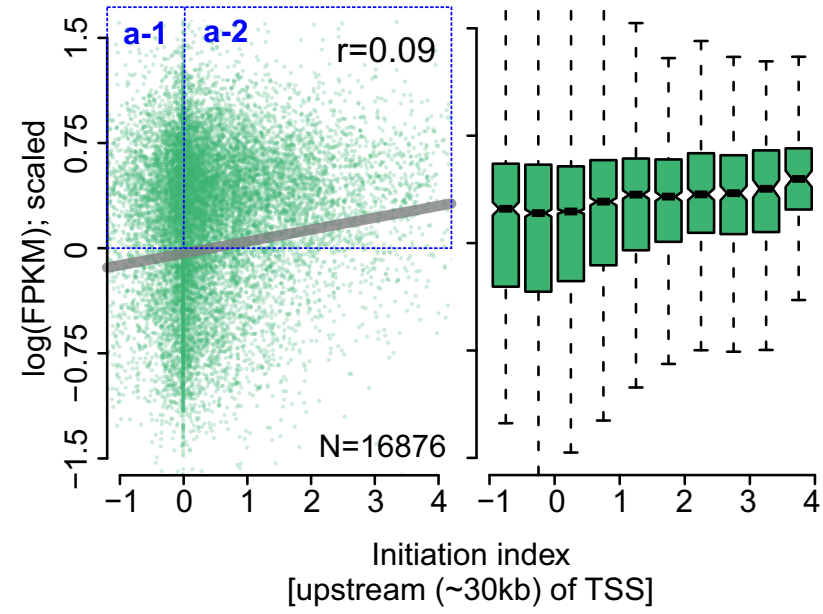

b

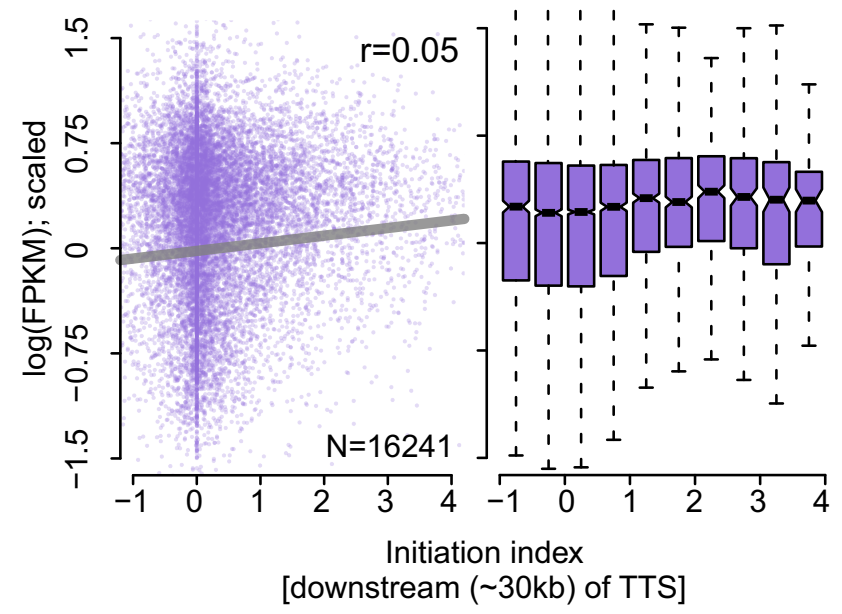

C

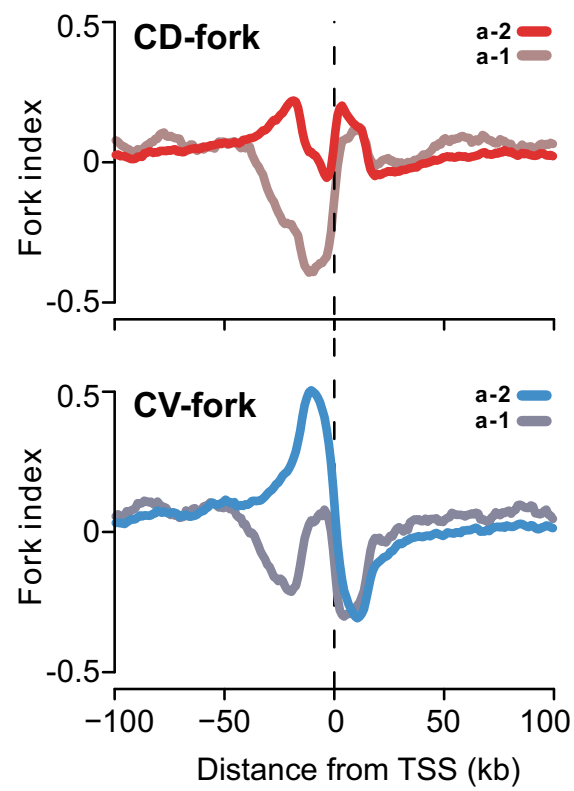

\section{Supplementary fig. 6 | Correlation of initiation and transcriptional activity.}

a. Top: Schematic of a gene showing TSS and TTS. Bottom: Sum of initiation index within the 0-30 kb region upstream of TSS (left two panels) and downstream of the TTS (two right panels) are plotted against transcriptional activity for each gene, represented as Fragments Per Kilobase of exon per Million mapped reads (FPKM) from an RNA-seq experiment (this study). Sum of initiation index and log(FPKM) are normalised to Z-score (average $=0$, standard deviation $=1$ ). $\mathbf{b}$. Venn diagrams representing the overlap between initiation zones, 0-30 kb upstream of TSS and 0-30 kb downstream of the TTS. c. Fork index across $\mathrm{a}+/-100 \mathrm{~kb}$ around annotated TTS in the human genome. Data of for the fork index around genes with $>$ average transcriptional activity are categorised into two groups as shown in (a): a-1, in which the sum of initiation index in the upstream of TSS is less than or equal to 0: average value $(n=2804)$, and the a-2, in which this value is greater than $0(n=11155)$. 


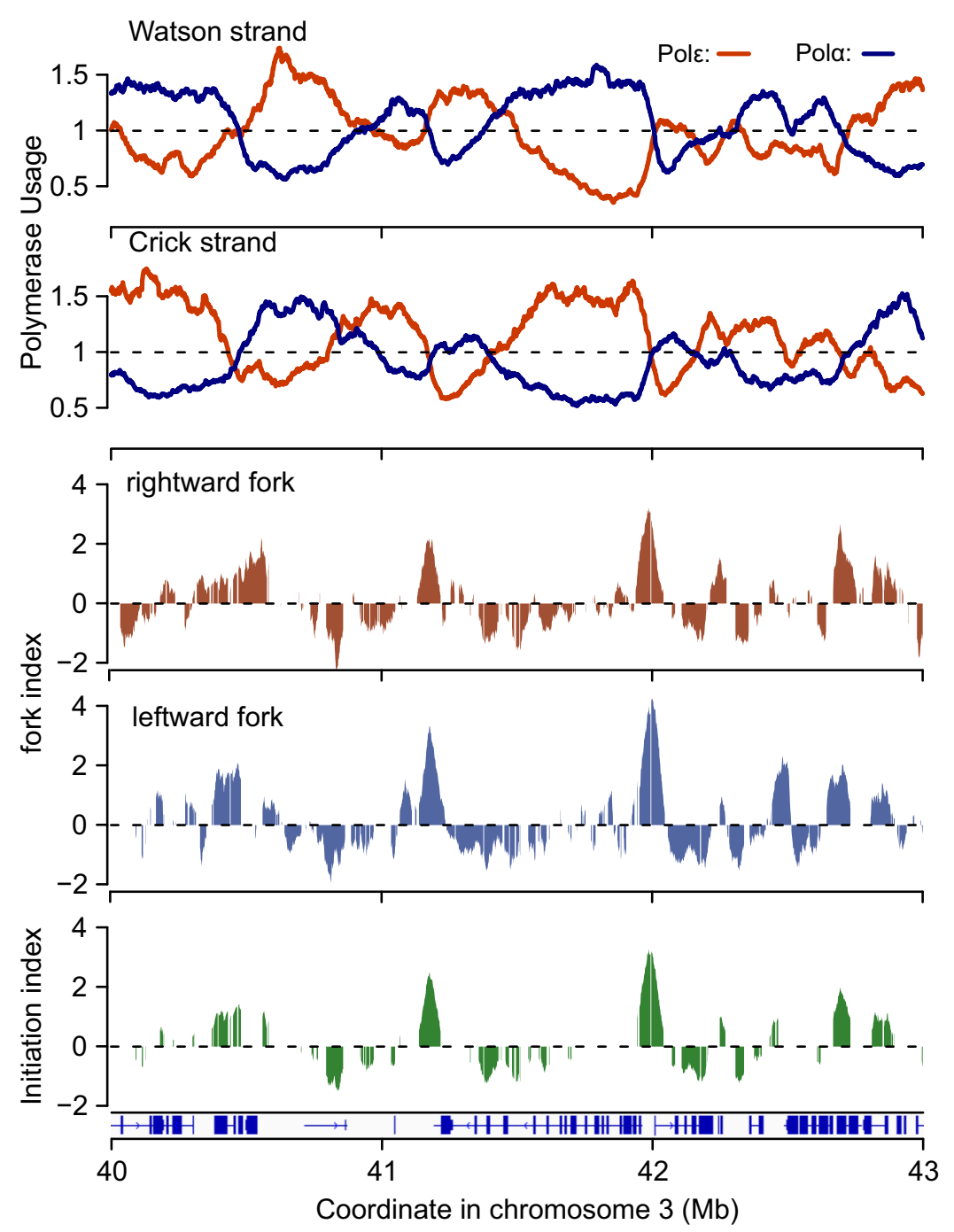

Supplementary fig.7 | The profiles of rightward and leftward forks in replicate 2.

Top: Profiles of polymerase usage plotted for a representative region of chromosome 3. Middle: leftward and rightward moving fork index $\left(\mathrm{Fk}^{R}\right.$ and $\left.\mathrm{Fk}^{L}\right)$ plotted for the same region. Bottom: initiation index plotted for the same region. Data used in these plots are derived from the experimental replicate 2 and are comparable to those from experimental replicate 1 that are used in Fig. 4b. 
a
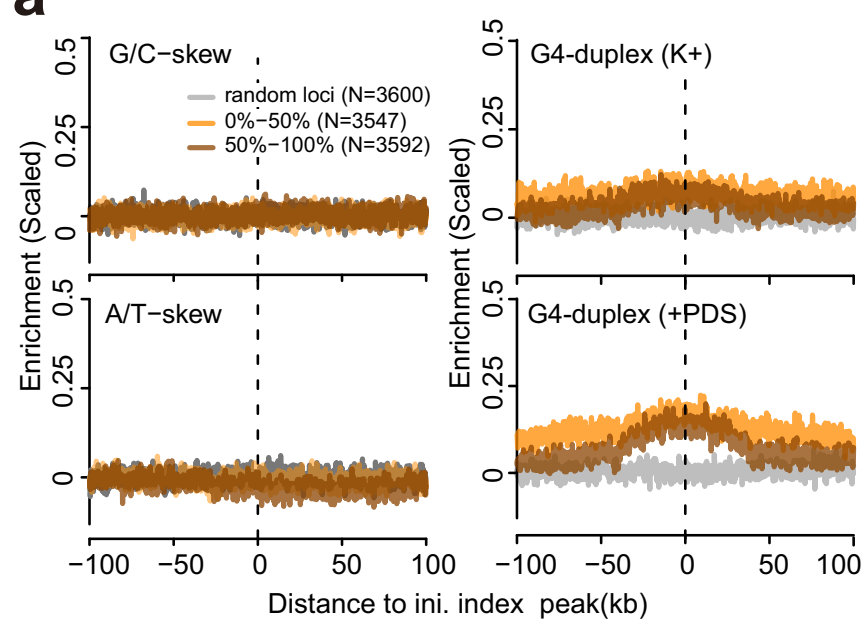

b

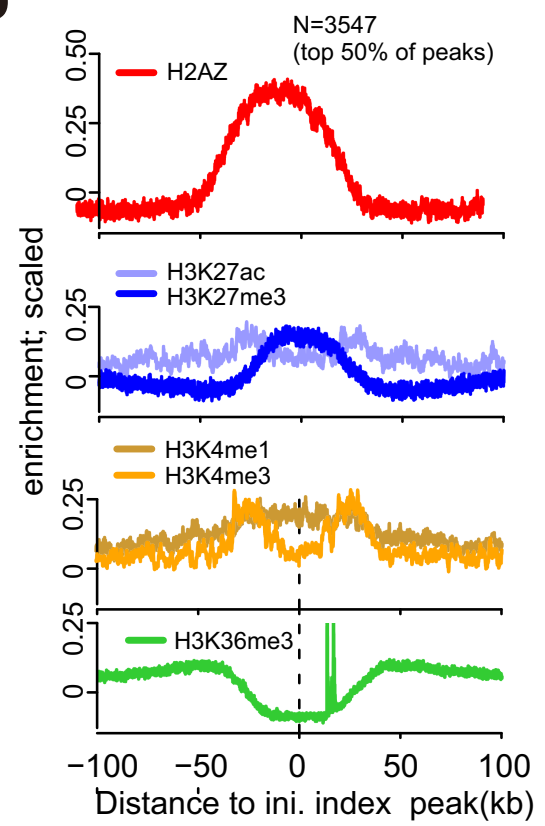

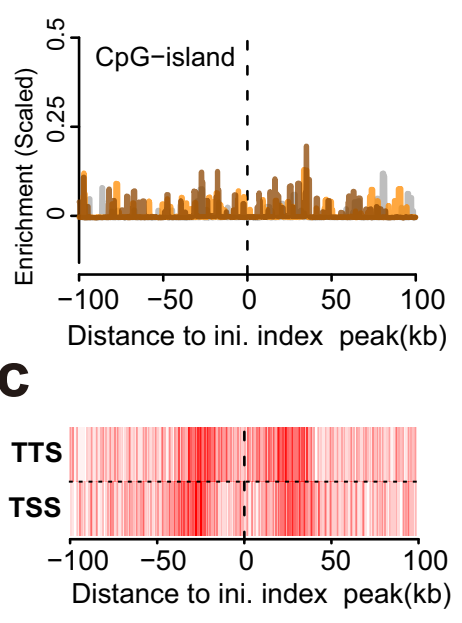

d

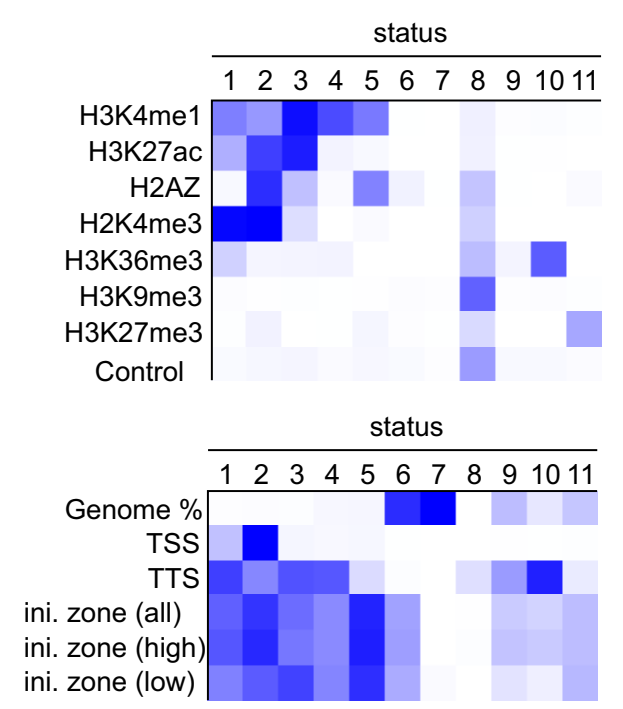

e
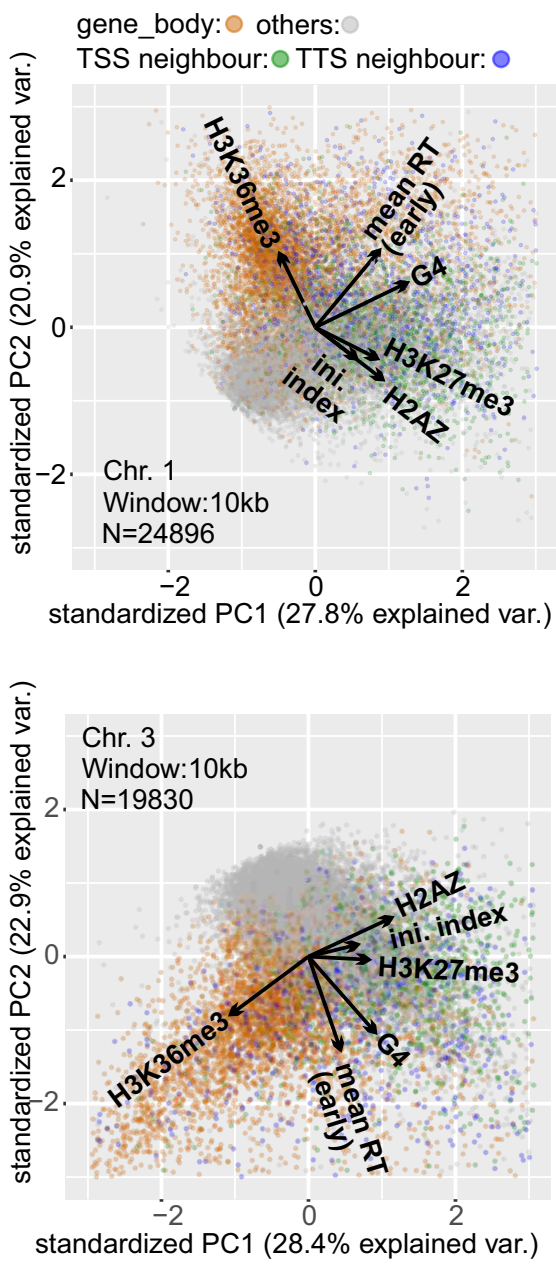

\section{Supplementary fig. 8 | Sequence features and chromatin status at replication initiation zones.}

a. Enrichment of sequence-based features G/C-skew, A/T-skew, G4-duplex and CpG-islands +/- $100 \mathrm{~kb}$ around the computationally detected peaks (yellow vertical lines in Extended Data Fig. 4d) in initiation zones that are concordantly detected in the two experimental replicates. Data for genome-wide G4-duplex formation are derived from G4-seq published in Marsico et al $(2019)^{50}$. K+; G4 duplexes formed in the presence of potassium ions. +PDS; G4 duplex formed in the presence of G4-targeting small molecule pyridostatin (PDS). b. Equivalent analysis for H2AZ, H3K27 acetylation (ac), H3K27 tri-methylation (me3), H3K4 mono-methylation (me1), H3K4me3 and H3K36me3. Histone modification data are derived from ChIP-seq data for HCT116 published in Lay et al $(2019)^{49}$ and these genome-wide data were z-score normalised before averaging around peaks of initiation index. c. Distribution of TSS and TTS around peaks of initiation index. d. Heat maps for the model of chromatin-state annotation produced by ChromHMM as described in Ernst and Kellis (2012) ${ }^{24}$. Top: the model was trained on the datasets indicated by the chromatin modification and defined 11 genomic status bins (status 1-11) that represent different chromatin states. The relative enrichments of chromatin features that are present in each status bin are represented by the extent of blue shading (for example, chromosome status bin 10 is enriched only for $\mathrm{H} 3 \mathrm{~K} 36 \mathrm{me}$, which distinguishes it from the remaining 10 bins). Bottom: the model then allocates the locations associated with the TSS, TTS and initiation zones to the same 11 bins (status 1-11), again representing the relative $\%$ occupancy by shade of blue (for example, most TSS are associated with bin 2. Referring back to the top heatmap reveals the correlating chromatin marks). e. Principal component analysis to generate a correlation map of initiation index scores with the indicated genomic features. Top: chromosome 1. Bottom: chromosome 3 . The arrows approximate the direction of each feature and their lengths approximate variances of the data. 

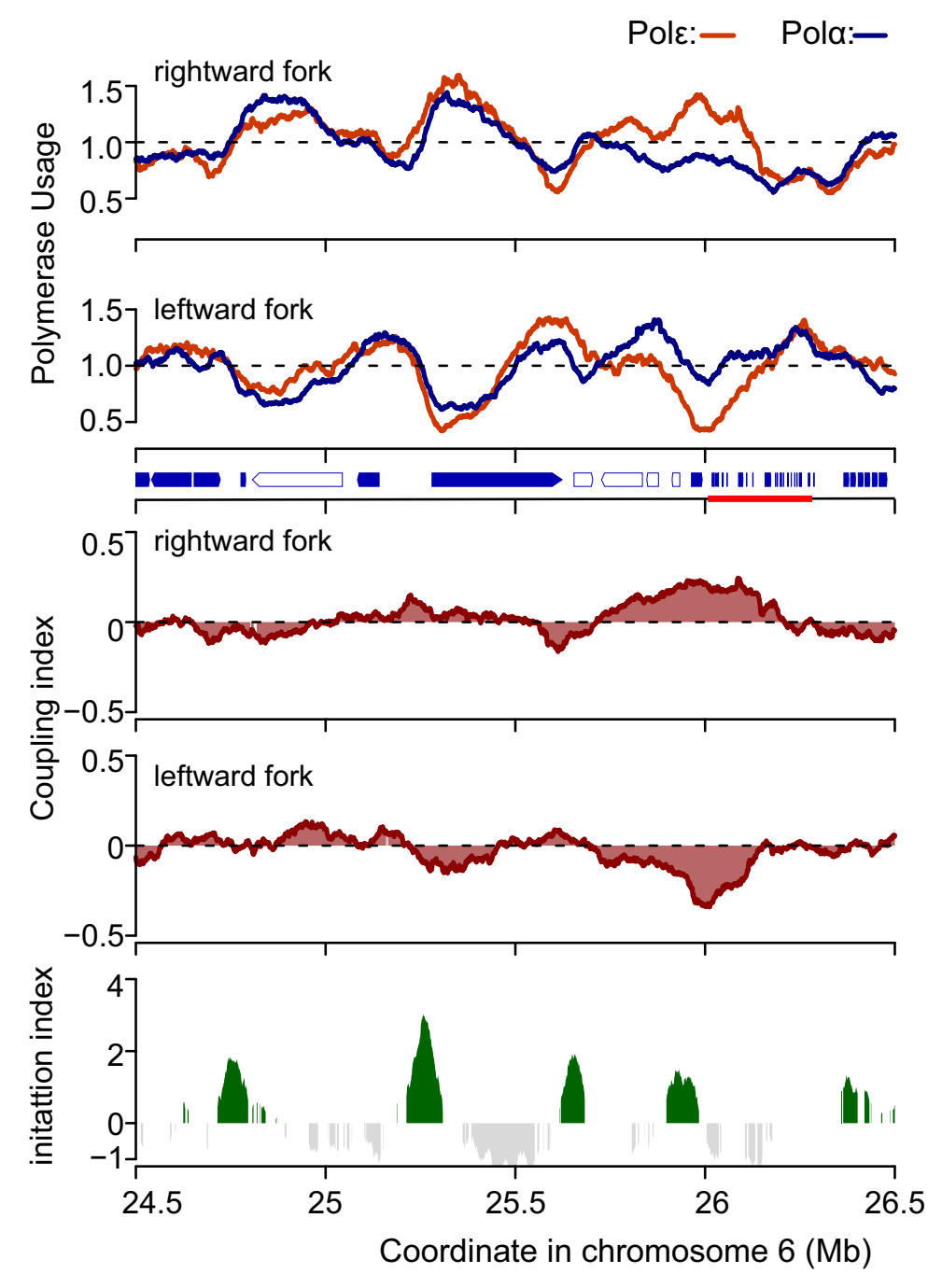

\section{Supplementary fig. 9 | DNA polymerase coupling/uncoupling in replicate 2.}

Equivalent data for replicate 2 as presented across the same representative region of chromosome 6 for replicate 1 in Fig. 5a,c. Top: Profiles of polymerase usage. Arrows on the vertical axis indicate active genes (blue-filled) and inactive genes (unfilled). The red line on the vertical axis indicates a cluster of histoneencoding genes. Middle: coupling index $(\mathrm{Cl})$ of rightward and leftward moving forks. Bottom: the initiation index of the same region for comparison. 
a

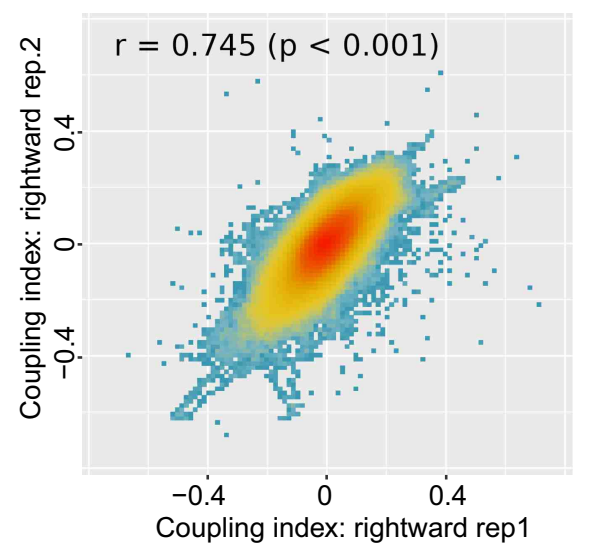

C

$\circ:$ population $\bigcirc$ : outliers (rigtward)
$\bigcirc:$ outliers (leftward) $\bigcirc:$ outliers (both)

$r=-0.357(p<0.001)$

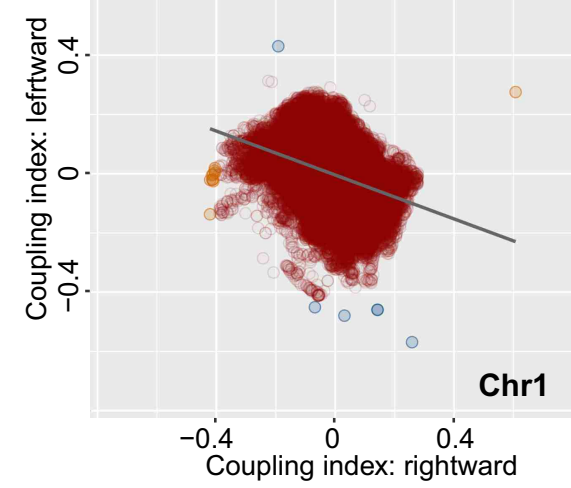

b
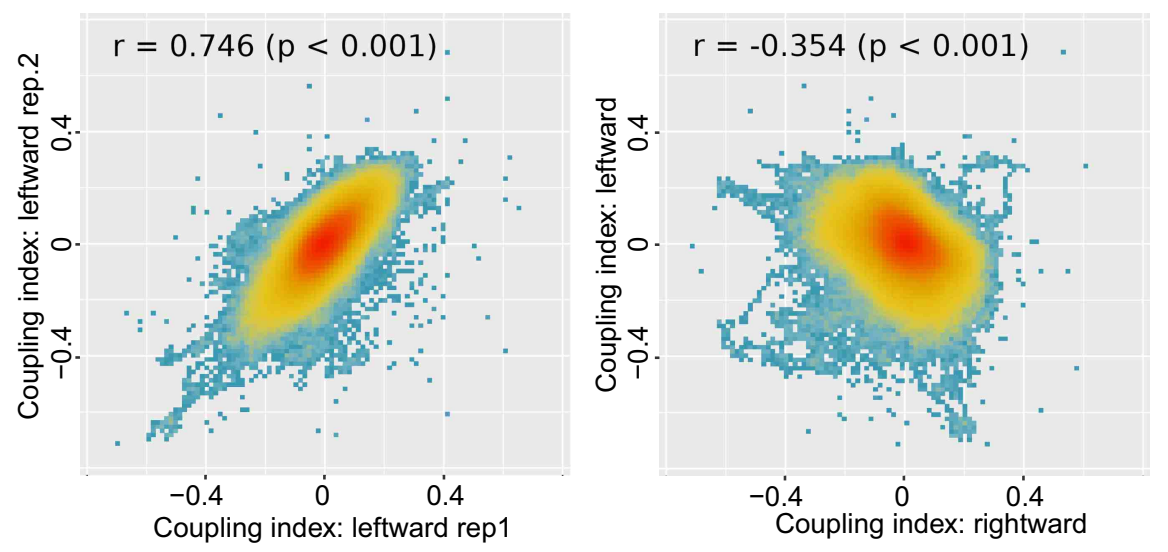

$$
r=-0.32(p<0.001)
$$

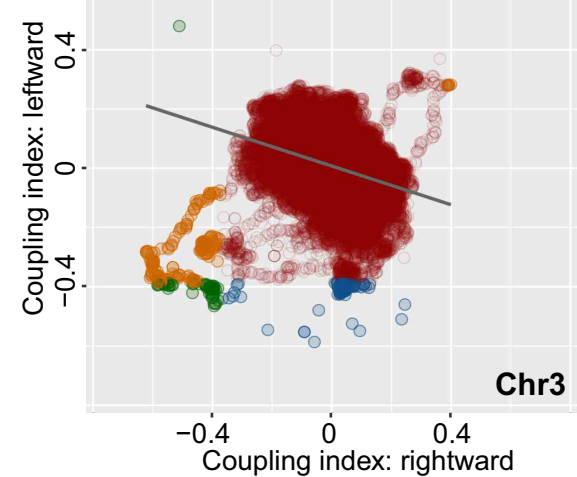

$$
r=-0.172(p<0.001)
$$

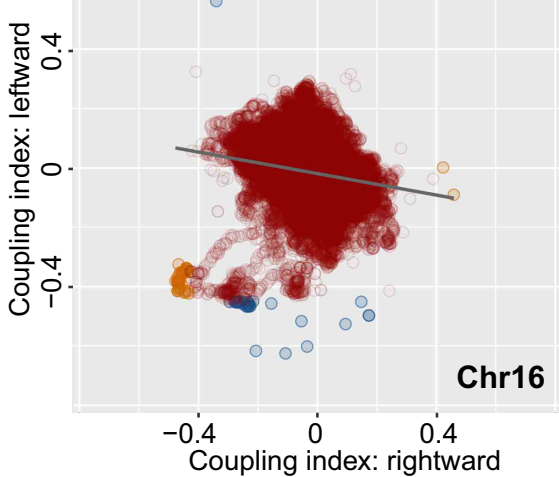

\section{Supplementary fig. 10 | The distribution of coupling index.}

a, correlation of coupling index $(\mathrm{Cl})$ derived from two experimental replicates. left: rightward moving forks, right: leftward moving forks. b. Correlation of $\mathrm{Cl}$ of rightward and leftward moving forks from replicate 2. See Fig. 5d for equivalent analysis for replicate 1. c. Correlation of $\mathrm{Cl}$ of rightward and leftward moving forks from replicate 2 presented by chromosome. Chromosomes 1, 3 and 16 are shown. Equivalent data for replicate 1 are shown in Fig. $6 a$. 